\title{
ON SOME MODEL PROBLEMS IN QUANTUM CONTROL*
}

\author{
NAVIN KHANEJA ${ }^{\dagger}$
}

\begin{abstract}
Control and manipulation of quantum mechanical systems using electromagnetic fields is a widely studied subject in areas of physics and chemistry, including spectroscopy, atomic molecular, and optical physics, and quantum chemistry. This article attempts to provide a glimpse into the rich class of bilinear control systems that are ubiquitous in these problems. In this article, we use control of spin systems in magnetic resonance as a model system to highlight characteristic feature of problems in quantum control. Background information is provided to enable the reader to appreciate new results and developments, where principled use of ideas from control theory have provided new insights into finding optimal ways to control and manipulate quantum mechanical systems. The study of deterministic and stochastic models that arise in problems in measurement and manipulation of quantum mechanical systems may foster new developments in control.
\end{abstract}

1. Introduction. The article describes some differential equation models that arise in the control and manipulation of quantum mechanical phenomena. Control of spin dynamics in NMR spectroscopy $[1,2,3]$ is used as a paradigm to outline general principles in the control of quantum systems and describe some common characteristic phenomenon encountered in control of these physical systems.

The defining equation for the state of a quantum mechanical system is the Schröedinger equation

$$
|\dot{\psi}\rangle=-i\left[H_{0}+\sum_{j=1}^{n} u_{j} H_{j}\right]|\psi\rangle
$$

where the state of the quantum system is represented by a vector $|\psi\rangle \in \mathcal{H}$, a suitable Hilbert space. $H_{0}$ and $H_{j}$ are Hermitian operators, representing Hamiltonians of the system and $u_{j}(t)$ are time varying functions that represent controls in the system dynamics. For models, discussed in this paper, $\mathcal{H}$ is finite dimensional and in a chosen basis, $H_{0}$ and $H_{j}$ are Hermitian matrices. We assume that $\mathcal{H}$ is finite, unless stated explicitly. Modulating $u_{j}$, effects the Hamiltonian of the system and therefore effects the evolution of the state of the system. Eq.(1) has the familiar form of a bilinear control system

$$
\dot{x}=\left(A+\sum_{j=1}^{n} u_{j} B_{j}\right) x
$$

where in the present context, $A, B_{j}$ are skew Hermitian matrices. The evolution preserves the norm of the state $|\psi\rangle$. The evolution is unitary and the state vector at

\footnotetext{
* Dedicated to Roger Brockett on the occasion of his 70th birthday.

${ }^{\dagger}$ To whom correspondence may be addressed. School of Engineering and Applied Sciences, Harvard University, Cambridge, MA 02138. E-mail: navin@hrl.harvard.edu
} 
time $t$ is related to initial state vector by an unitary transformation $U(t)$, such that

$$
|\psi(t)\rangle=U(t)|\psi(0)\rangle
$$

where $U(t)$ satisfies the differential equation

$$
\dot{U}=-i\left[H_{0}+\sum_{j=1}^{n} u_{j} H_{j}\right] U, \quad U(0)=\mathbf{1}
$$

Where $\mathbf{1}$ is the identity matrix. A textbook example of a system, where such a bilinear control model arises is the evolution of the magnetic moment of a spin $\frac{1}{2}$ in a magnetic field $B$. Spin, like charge is a physical property of elementary particles. It is a measure of their intrinsic angular momentum and the state of a spin $\frac{1}{2}$ is represented by a complex vector of dimension 2. The Hamiltonian generates rotations on the state space of a quantum system. The Hamiltonian of a spin $\frac{1}{2}$ can be written in terms of the generators of rotations on a two dimensional space and these are the Pauli matrices $-i \sigma_{x},-i \sigma_{y},-i \sigma_{z}$, where,

$$
\sigma_{z}=\frac{1}{2}\left[\begin{array}{cc}
1 & 0 \\
0 & -1
\end{array}\right] ; \quad \sigma_{y}=\frac{1}{2}\left[\begin{array}{cc}
0 & -i \\
i & 0
\end{array}\right] ; \quad \sigma_{x}=\frac{1}{2}\left[\begin{array}{cc}
0 & 1 \\
1 & 0
\end{array}\right] \text {. }
$$

Note

$$
\left[\sigma_{x}, \sigma_{y}\right]=i \sigma_{z}, \quad\left[\sigma_{y}, \sigma_{z}\right]=i \sigma_{x}, \quad\left[\sigma_{z}, \sigma_{x}\right]=i \sigma_{y}
$$

where $[A, B]=A B-B A$ is the matrix commutator and

$$
\sigma_{x}^{2}=\sigma_{y}^{2}=\sigma_{z}^{2}=\frac{\mathbf{1}}{4}
$$

The classical energy $E$ of the magnetic moment $\mu$ of a spin in a magnetic field is $E=-\mu \cdot B$. The magnetic moment of a spin is proportional to its angular momentum, given by $\mu=\gamma L$, where $\gamma$ is the gyromagnetic ratio (a characteristic property of the nucleus) and $L$ is the angular momentum operator. Therefore, the Hamiltonian of $\operatorname{spin} \frac{1}{2}$ is

$$
H=-\gamma\left[B_{x} L_{x}+B_{y} L_{y}+B_{z} L_{z}\right]
$$

where $L_{x}, L_{y}, L_{z}$ are now operators, representing angular momentum in the $x, y, z$ direction respectively.

Since angular momentum is generator of rotation, the angular momentum operators $L_{x}, L_{y}, L_{z}$ are identified with the Pauli matrices $\sigma_{x}, \sigma_{y}, \sigma_{z}$, the generators of rotation in a two dimensional Hilbert space. The Schröedinger equation then takes the form

$$
|\dot{\psi}\rangle=i \gamma\left[\sigma_{z} B_{0}+\sigma_{y} B_{y}(t)+\sigma_{x} B_{x}(t)\right]|\psi\rangle,
$$




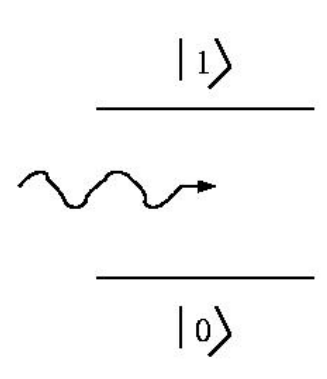

A

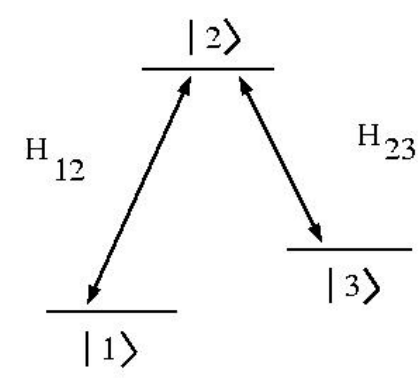

B

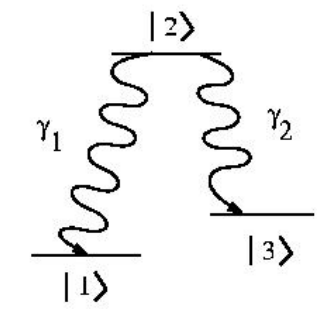

$\mathrm{C}$

FIG. 1. The Fig. A shows the simplest of the quantum objects, a two level system being probed with an electromagnetic field. Fig. B shows energy level diagram of a three level Lambda system often studied in the area of Laser spectroscopy. Fig. C shows spontaneous decay of the population in state $|2\rangle$ to energy levels $|1\rangle$ and $|3\rangle$.

where, we use $B_{0}=B_{z}$. Let $\omega_{0}=\gamma B_{0}$ and $(u(t), v(t))=\left(\gamma B_{x}(t), \gamma B_{y}(t)\right)$. The above equation is then rewritten as

$$
|\dot{\psi}\rangle=\frac{i}{2}\left[\begin{array}{cc}
\omega_{0} & u-i v \\
u+i v & -\omega_{0}
\end{array}\right]|\psi\rangle
$$

The eigenstates of $\sigma_{z}$, labeled, $|0\rangle=\left[\begin{array}{l}1 \\ 0\end{array}\right]$ and $|1\rangle=\left[\begin{array}{l}0 \\ 1\end{array}\right]$, with eigenvalues $\frac{1}{2}$ and $-\frac{1}{2}$, correspond to the state of the spins oriented along or opposite to the magnetic field $B_{0}$. Eq. (9) represents the most basic of all quantum mechanical objects, a two level system being manipulated by an external field. A schematic of such a system is shown in Fig. 1A.

The differential equation model (9) describes dynamics of spin $\frac{1}{2}$ in the nuclear magnetic resonance (NMR) experiments when manipulated by transverse magnetic fields $\left(B_{x}(t), B_{y}(t)\right)$ which manifest themselves as control inputs $(u(t), v(t))$. Primary goal of these experiments is to accurately measure $\omega_{0}$ by manipulating or probing the system with control inputs $(u(t), v(t))$, which provides a wealth of information on chemistry and structure of molecules carrying spins, as detailed subsequently. NMR experiments are performed on an ensemble of spins. All the members of the ensemble may not have identical state vectors. In which case, the description of a quantum system is a density matrix as described by

$$
\rho=\sum_{j} p_{j}\left|\psi_{j}\right\rangle\left\langle\psi_{j}\right|
$$

where $p_{j}$ is the proportion of ensemble elements $\left(\sum_{j} p_{j}=1\right)$ in the state $\left|\psi_{j}\right\rangle$ (The notation $\left\langle\psi_{j} \mid \psi_{j}\right\rangle$ and $\left|\psi_{j}\right\rangle\left\langle\psi_{j}\right|$, denote inner and outer product of vector $\left|\psi_{j}\right\rangle$ with itself respectively). In an ensemble of spin $\frac{1}{2}$, with $\frac{1}{3}$ of spins in the state $|0\rangle$ and $\frac{2}{3}$ 
of spins in the state $|1\rangle$, the density matrix

$$
\rho=\left[\begin{array}{cc}
\frac{1}{3} & 0 \\
0 & \frac{2}{3}
\end{array}\right] .
$$

By the postulates of quantum mechanics, the observable quantities are represented by self-adjoint operators. The expected value of an observable $\mathbf{O}$, when the quantum system is in the state $|\psi\rangle$ is simply $\langle\psi|O| \psi\rangle$, where $O$ is the operator that represents the observable $\mathbf{O}$. Therefore, for an ensemble of quantum systems, the expected value of an observable $\mathbf{O}$ is

$$
\langle O\rangle=\sum_{j} p_{j}\left\langle\psi_{j}|O| \psi_{j}\right\rangle=\operatorname{tr}(\rho O)
$$

The density matrix $\rho$ of the quantum system evolves as

$$
\dot{\rho}=-i\left[H_{0}+\sum_{j=1}^{n} u_{j} H_{j}, \rho\right],
$$

where $[A, B]$, as before is the matrix commutator. This follows from simply differentiating the Eq. (10), where each $\left|\psi_{j}\right\rangle$ satisfies the same Eq. (1). Some properties of the density matrix $\rho$ are evident from its construction. It is a Hermitian, positive semidefinite and satisfies $\operatorname{tr}(\rho)=1$. The evolution of the density operator is

$$
\rho(t)=U(t) \rho(0) U^{\dagger}(t)
$$

where $U(t)$ is the unitary transformation in (4). Also by construction, $\operatorname{tr}\left(\rho^{2}\right) \leq 1$, with equality holding only if only one of the $p_{j}$ in Eq. (10) is non-zero and equal to 1. For an ensemble of spin $\frac{1}{2}$, the density matrix $\rho$ is a $2 \times 2$, Hermitian matrix, which can be decomposed as

$$
\rho=\frac{1}{2} \mathbf{1}+m_{x} \sigma_{x}+m_{y} \sigma_{y}+m_{z} \sigma_{z}
$$

Therefore, for the density matrix in Eq. (13), we obtain that the expected value of the angular momentum along $x, y, z$, represented by operators $\sigma_{x}, \sigma_{y}$ and $\sigma_{z}$ is then simply proportional to $m_{x}, m_{y}, m_{z}$. Then, Eq. (9) implies

$$
\frac{d}{d t} \underbrace{\left[\begin{array}{l}
m_{x} \\
m_{y} \\
m_{z}
\end{array}\right]}_{M}=\left[\begin{array}{ccc}
0 & -\omega_{0} & v(t) \\
\omega_{0} & 0 & -u(t) \\
-v(t) & u(t) & 0
\end{array}\right]\left[\begin{array}{l}
m_{x} \\
m_{y} \\
m_{z}
\end{array}\right],
$$

where the vector $M=\left(m_{x}, m_{y}, m_{z}\right)^{\prime}$ is a measure of the net magnetic moment or magnetization of the spin ensemble and the above equation is the well studied Bloch equation, which describes the precession of the magnetic moment in a magnetic field 
and can be concisely written as $\dot{M}=\gamma M \times B$, where $B=\left(B_{x}(t), B_{y}(t), B_{0}\right)^{\prime}$ is the magnetic field vector as defined before. Observe, Eq. (14) evolves on a sphere and for future, we normalize the norm of $M$ to 1. Equation (14) is at the heart of subject of NMR spectroscopy, where a typical task is to engineer $(u(t), v(t))$ to manipulate or steer the vector $M$ in order to estimate the parameter $\omega_{0}$. In the following subsection, we describe some characteristic feature of the control inputs for the manipulation of Eq. (14).

1.1. Control of Bloch Equations. Note, Eq. (14), can be concisely written as

$$
\dot{M}=\left(\omega_{0} \Omega_{z}+u(t) \Omega_{x}+v(t) \Omega_{y}\right) M,
$$

where

$$
\Omega_{x}=\left[\begin{array}{ccc}
0 & 0 & 0 \\
0 & 0 & 1 \\
0 & -1 & 0
\end{array}\right], \Omega_{y}=\left[\begin{array}{ccc}
0 & 0 & 1 \\
0 & 0 & 0 \\
-1 & 0 & 0
\end{array}\right], \Omega_{z}=\left[\begin{array}{ccc}
0 & -1 & 0 \\
1 & 0 & 0 \\
0 & 0 & 0
\end{array}\right] .
$$

A typical control problem is to steer the system from its equilibrium state $M(0)=$ $(0,0,1)$ to a terminal state on the equator. A salient feature of such problems is that the external excitations $(u(t), v(t))$ are typically, significantly smaller in strength as compared to the natural dynamics represented by $\omega_{0}$, which is four to five orders of magnitude larger in the NMR experiments. Therefore, for the external control to be effective in manipulating the system, it is essential that the control be oscillatory. To fix ideas, let

$$
(u(t), v(t))=A(t)\left(\cos \left(\omega_{1} t+\theta(t)\right), \quad \sin \left(\omega_{1} t+\theta(t)\right)\right),
$$

and consider the control problem of steering $M$ in the Eq. (15) from an initial point $(0,0,1)^{\prime}$ to the final target state $(1,0,0)^{\prime}$. Observe, by transforming to a coordinate system such that $X=\exp \left(-\omega_{1} \Omega_{z} t\right) M$, we obtain that

$$
\dot{X}=\left(\omega \Omega_{z}+u_{1}(t) \Omega_{x}+v_{1}(t) \Omega_{y}\right) X,
$$

where $\omega=\omega_{0}-\omega$, and $\left(u_{1}(t), v_{1}(t)\right)=(A(t) \cos \theta(t), A(t) \sin \theta(t))$. Now, since $X(0)=$ $M(0)$, by simply choosing $\omega=\omega_{0}, \theta(t)=\theta$ and $A(t)=A$, both constant, we drive $X(0)$ to $(\sin \theta,-\cos \theta, 0)$ in $T=\frac{\pi}{2 A}$ units of time. When $\omega=\omega_{0}$, equation (18) has no natural dynamics. Therefore, it doesn't matter how weak $A(t)$ is, given sufficient time, $X(0)$ can be steered to the transverse plane. $X(T)$ can be put anywhere on the transverse plane by appropriate choice of $\theta$. Left panel in Fig. 2 shows this transfer, both in the rotating frame as in Eq. (18), (the blue curve) and the laboratory frame Eq. (15) (red curve). However, a choice of constant control $(u(t), v(t))=$ 
( $A \cos \theta, A \sin \theta$ ), would be completely ineffective, if applied to the original system in Eq. (15), as the net motion is then a precession around the axis $\left(A \cos \theta, A \sin \theta, \omega_{0}\right)$, as shown in Fig. 2 and therefore significantly falls short of the desired transfer when $A \ll \omega_{0}$. Therefore, it is desirable to use a control input as in Eq. (17), which is oscillatory at the frequency of natural oscillation of the system. It is not difficult to show that the choice of such a control input is the minimum energy control for driving the system in Eq. (15) to the transverse plane. Applying such a control input corresponds to exciting the system at its resonance and with a very weak field, the system can be driven far from equilibrium. $A(t), \theta(t)$ and $\omega_{0}$ are naturally termed amplitude, phase and carrier frequency of the applied radio-frequency field as in radio communication. Design of appropriate control inputs $(u(t), v(t))$ is infact the design of an appropriate amplitude and phase modulations.
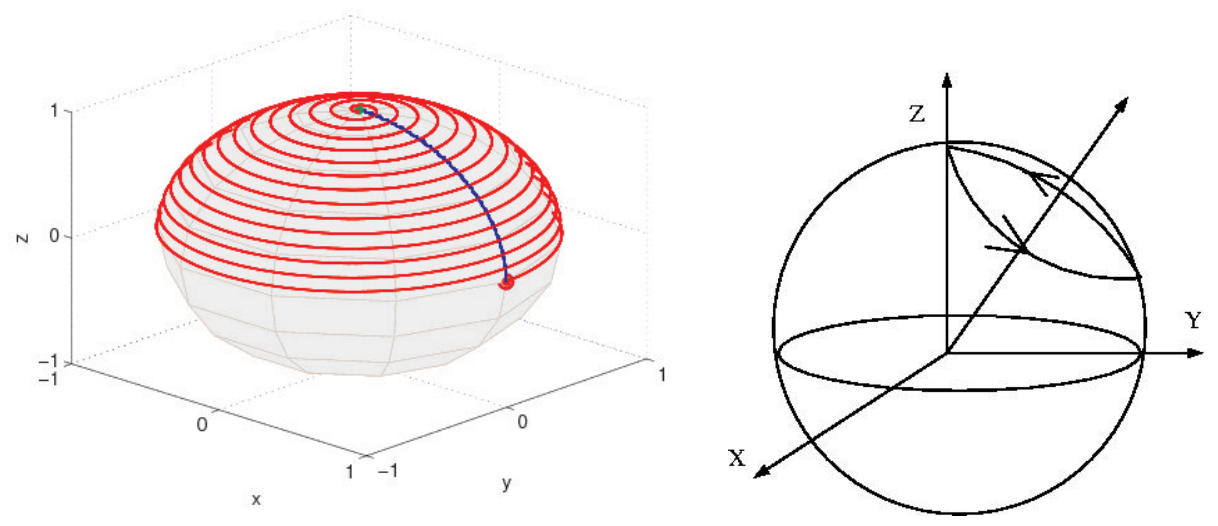

FIG. 2. The figure shows the rotation of the vector $X$ in Eq. (18), around a tilted axis, when $\Delta \omega$ is comparable to strength of control $A$.

After the magnetic moment is driven to the transverse plane by choice of an appropriate control input, the oscillating control is switched off and the magnetic moment $M$ precesses around the static magnetic field $B_{0}$ with a frequency $\omega_{0}$. This is just the evolution in Eq. (17), after the controls are switched off. This precessing magnetic moment by Faraday's law induces an oscillating current in the nearby placed receiver coil and is termed as free induction decay (FID) ( Top right of Fig. 3 shows the FID ). This FID, when Fourier transformed, shows a peak at $\omega_{0}$. At the magnetic field strength of $B_{0}=14$ Tesla, $\omega_{0}$ for hydrogen nuclei is $600 \mathrm{Mhz}$, for carbon, 150 Mhz and for nitrogen, $60 \mathrm{Mhz}$. The frequency $\omega_{0}$ of an atomic nuclei is also dependent on its chemical/electronic environment in a molecule. The secondary magnetic fields produced on an atomic nuclei by its electronic environment results in a shift of the frequency $\omega_{0}$ to $\omega_{0}(1-\sigma)$, where $\sigma$ is specific to the chemical environment of the nuclear spins and is usually of the order $10^{-6}$, so when $\omega_{0}$ is around $500 \mathrm{Mhz}$, 


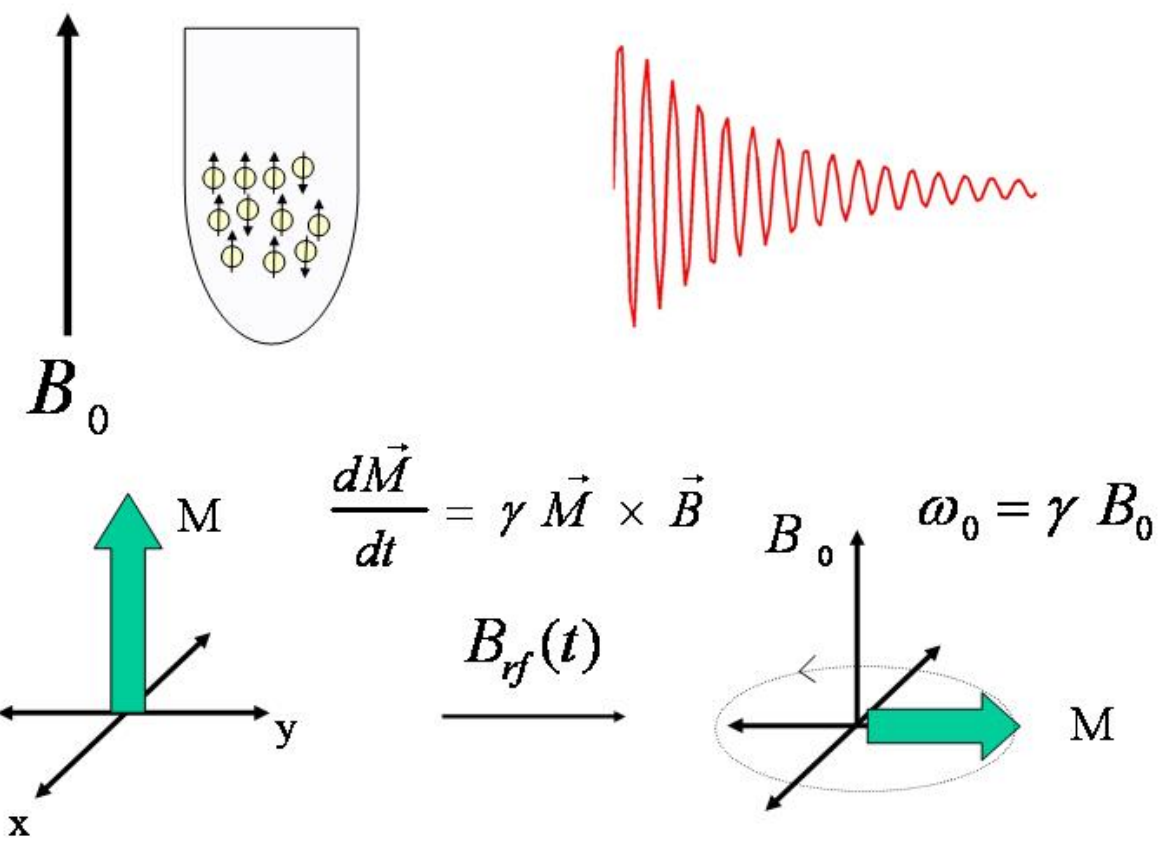

FIG. 3. The top figure shows the basic features of an NMR experiment. The top left of part of the figure depicts use of a field $B_{0}$ to polarize the sample. The bottom figure shows use of pulsed magnetic fields to steer the net magnetization and generate FID. The top left panel shows the profile of a free induction decay.

the shift $\omega_{0} \sigma$ is in Khz. The Fourier transform of the FID signal then shows many peaks, corresponding to different nuclei with their chemical environment specific characteristic shifts. Fig. 4 shows a typical proton NMR spectra from two different size molecules. NMR is therefore an important analytical tool in chemistry as the peaks in the NMR spectrum serve as a characteristic finger print of the molecule. Starting as a tool for characterization of organic molecules, the use of NMR has spread to areas as diverse as pharmaceutics, medical diagnostics (medical resonance imaging) and structural biology $[4,5]$. The principles of NMR have served as a paradigm for other physical methods that rely on interaction between radiation and matter. It is therefore not surprising that experiments in NMR also serve as good model problems in control of quantum systems.

Eq. (17) gives the wrong impression that the magnetic moment on the transverse plane will continue to precess for ever. Overtime, the magnetic moments of spins making the magnetization vector $M$ experience local fluctuations in the ambient field $B_{0}$, causing them to precess differently and hence lose coherence (decoherence). This gives the FID, a decaying envelop (see Fig. 4). This phenomenon, termed decoherence 


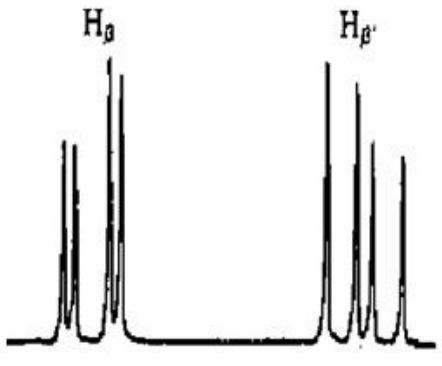

A

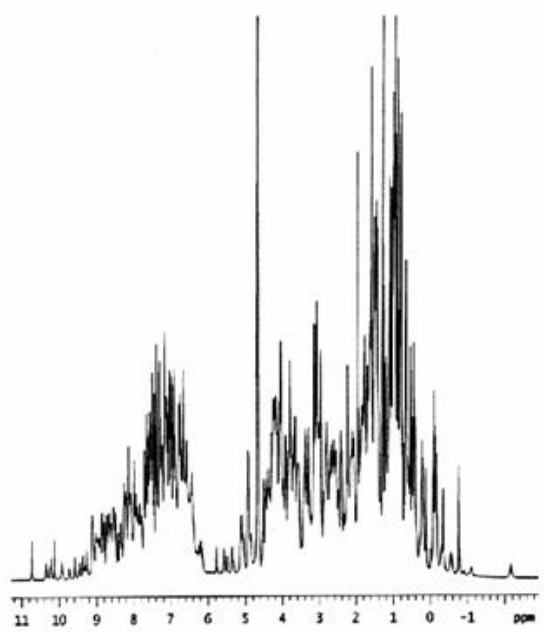

$750 \mathrm{MHz}$ spectrum of the protein lysozyme

$\mathrm{B}$

FIG. 4. The figure shows a typical proton NMR spectra of a small and medium sized molecule, shown in left and right panel respectively.

is described in detail subsequently and explicit models are derived to analyze the effect. This leads to the study of open quantum systems where a quantum system interacts with external environment but one is only interested in the dynamics of the quantum system of interest. Additional terms need to be incorporated in Eq. (11) to account for this effect. Before we describe dynamics of open quantum systems, few general comments about oscillatory control described before are in order.

The oscillatory control described in Eq. (17) consists of irradiating the spin ensemble with an oscillating field along the $x$ and $y$ direction. In practice, the same effect can be obtained with simply having a single oscillating field along say $x$ direction. This corresponds to

$$
(u(t), v(t))=(2 A(t) \cos (\omega t+\theta), 0) .
$$

Observe, such a control can be written as superposition of two control inputs $\left(u_{1}, v_{1}\right)=$ $A(t)\left(\cos \left(\omega_{0} t+\theta\right), \sin \left(\omega_{0} t+\theta\right)\right)$ and $\left(u_{2}, v_{2}\right)=A(t)\left(\cos \left(\omega_{0} t+\theta\right),-\sin \left(\omega_{0} t+\theta\right)\right)$. Transforming again into a rotating frame results in the equation

$$
\dot{X}=\left(A(t)\left(\cos \theta+\cos \left(2 \omega_{0} t+\theta\right)\right) \Omega_{x}+A(t)\left(\sin \theta-\sin \left(2 \omega_{0} t+\theta\right)\right) \Omega_{y}\right) X .
$$

Since $\omega_{0} \gg A(t)$, the oscillating terms average out, giving identical equation as in Eq. 
(18). This averaging of the fast oscillating terms is often termed the rotating wave approximation.

1.2. Oscillatory Control. The characteristic nature of control inputs described in the previous section is the oscillatory character. In general, consider a $n$ dimensional quantum system where the control $u$ is used to modulate the Hamiltonian $H_{1}$ in

$$
\dot{\rho}=-i\left[H_{0}+u H_{1}, \rho\right]
$$

Now, since $H_{0}$ is a Hermitian operator, let $\left|\phi_{j}\right\rangle$ denote the orthogonal eigenvectors, with eigenvalues $\omega_{j}$. Then $\left|\phi_{j}\right\rangle$ diagonalize $H_{0}$, i.e, we rewrite $H_{0}=\sum_{j} \omega_{j}\left|\phi_{j}\right\rangle\left\langle\phi_{j}\right|$ and then note $\left|\phi_{j}\right\rangle\left\langle\phi_{j}\right|$ all commute and $\exp \left(-i \omega_{j}\left|\phi_{j}\right\rangle\left\langle\phi_{j}\right| t\right)=\exp \left(-i \omega_{j} t\right)\left|\phi_{j}\right\rangle\left\langle\phi_{j}\right|$, implying that

$$
\exp \left(-i H_{0} t\right)=\sum_{j} \exp \left(-i \omega_{j} t\right)\left|\phi_{j}\right\rangle\left\langle\phi_{j}\right|
$$

Now, transforming Eq. (19) into a rotating frame

$$
\rho_{r}=\exp \left(i H_{0} t\right) \rho \exp \left(-i H_{0} t\right)
$$

gives

$$
\dot{\rho}_{r}=-i\left[u(t) \exp \left(i H_{0} t\right) H_{1} \exp \left(-i H_{0} t\right), \rho_{r}\right]
$$

Now, let $h_{j k}=\left\langle\phi_{j}\left|H_{1}\right| \phi_{k}\right\rangle$. This, then gives that

$$
u(t) \exp \left(i H_{0} t\right) H_{1} \exp \left(-i H_{0} t\right)=u(t) \sum_{j k} h_{j k} \exp \left(-i \omega_{j k} t\right)|j\rangle\langle k|
$$

where $\omega_{j k}=\omega_{k}-\omega_{j}$ and we assume $\omega_{j k}$ are all distinct, such that $|u| \leq\left|\omega_{j k}-\omega_{l m}\right|$. If $u$ is modulated at one of the $\omega_{j k}$, i.e., $u(t)=A(t) \cos \left(\omega_{j k} t+\theta(t)\right)$, where the variation in $A(t)$ and $\theta(t)$ is assumed to be much slower than $\omega_{j k}$, then the resulting Hamiltonian in Eq. (19) averages to

$$
\dot{\rho_{r}}=-i \frac{A}{2}\left[h_{j k} \exp (i \theta(t))\left|\phi_{j}\right\rangle\left\langle\phi_{k}\left|+\exp (-i \theta(t)) h_{k j}\right| \phi_{k}\right\rangle\left\langle\phi_{j}\right|, \rho_{r}\right]
$$

where $h_{j k}=h_{k j}^{*}$. By modulating the Hamiltonian at the frequency of the difference of the energies of the eigenstates $\left|\phi_{k}\right\rangle$ and $\left|\phi_{j}\right\rangle$, we obtain effective Hamiltonians

$$
\begin{aligned}
& H_{j k}=h_{j k}\left|\phi_{j}\right\rangle\left\langle\phi_{k}\left|+h_{k j}\right| \phi_{k}\right\rangle\left\langle\phi_{j}\right|, \\
& G_{j k}=-i h_{j k}\left|\phi_{j}\right\rangle\left\langle\phi_{k}\left|+i h_{k j}\right| \phi_{k}\right\rangle\left\langle\phi_{j}\right|
\end{aligned}
$$

which induces a transition from state $j$ to state $k$ and vice versa. If all $\omega_{j k}$ are distinct, then we can assume that one can synthesize Hamiltonians $H_{j k}$, independently by simply choosing the frequency of the control $u(t)$. Therefore, one can write an effective control system for (19), which takes the form 


$$
\dot{\rho}_{r}=-i\left[\sum_{j k} u_{j k} H_{j k}+v_{j k} G_{j k}, \rho_{r}\right]
$$

where $u_{j k}, v_{j k}$ are controls that can be turned on and off. Some of $h_{j k}$ and therefore $H_{j k}$ might be zero and hence $H_{1}$ cannot induce a transition between the eigenstates $\left|\phi_{j}\right\rangle$ and $\left|\phi_{k}\right\rangle$ directly. These constraints are often termed as the selection rules in physics. Fig. 1 shows the energy level diagram of so called Lambda system studied in Laser Spectroscopy. There is no direct transition between states $|1\rangle$ and $|3\rangle$, but there is an indirect transition through the state $|2\rangle$.

Of fundamental interest is to know if the system in Eq. (4) can be driven between states of interest. This is the standard problem of controllability of bilinear systems. Therefore, the standard techniques $[6,7,8]$ for studying controllability of systems evolving on compact Lie groups can be directly applied [12]. The main result being that if the Lie algebra $\left\{-i H_{0},-i H_{j}\right\}_{L A}$, spanned by $\left\{-i H_{0},-i H_{j}\right\}$, is the Lie algebra $s u(n)$, where $n$ is the state space of the system in Eq. (4), then the system is controllable. Then, checking for controllability reduces to checking the Lie algebraic rank condition. For example, although there is no direct transition between states $|1\rangle$ and $|3\rangle$ in Fig. 1B, the system is controllable. The unitary propagator for the effective control system in Eq. (24), evolves as

$$
\dot{U}=-i\left[\begin{array}{ccc}
0 & \Omega_{c}(t) & 0 \\
\Omega_{c}^{*}(t) & 0 & \Omega_{p}(t) \\
0 & \Omega_{p}^{*}(t) & 0
\end{array}\right] U
$$

where $\Omega_{c}(t)$ and $\Omega_{p}(t)$ are complex valued controls that induce transitions between $|1\rangle$ and $|2\rangle$ and $|3\rangle$ and $|2\rangle$ respectively. The subject of explicit synthesis of the control laws for control of Eq. (4) has received significant attention recently in context of control of spin systems $[9,10,11]$. We will discuss some of these results subsequently.

\section{Open Quantum Systems.}

2.1. Master Equations. Eq. (11) describes the evolution of a closed quantum system. We now derive an equation for the dynamics of the open quantum systems. The derivation is not the most general, but captures the essence of how such a model is usually arrived at $[3,13,14]$. The effect of the environment on the system is modeled by an Hamiltonian $H_{1}$, which randomly fluctuates with time.

$$
\dot{\rho}=-i\left[H_{0}+f(t) H_{1}, \rho\right] .
$$

The correlation time of these fluctuations is assumed to be significantly shorter than the fastest time scale in the system dynamics. Therefore, we model these fluctuations 
as a white noise process such that $E[f(t+\tau) f(t)]=k \delta(\tau)$, where $\delta(\tau)$ is the Diracdelta function. Then, we have

$$
\rho(\tau)=U(\tau) \rho(0) U^{\dagger}(\tau)
$$

where we retain only terms of the second order in the expansion of $U$, which gives

$U(\tau)=I-i \int_{0}^{\tau}\left(H_{0}+f(\sigma) H_{1}\right) d \sigma-\int_{0}^{\tau}\left(H_{0}+f\left(\sigma_{1}\right) H_{1}\right) \int_{0}^{\sigma_{1}}\left(H_{0}+f\left(\sigma_{2}\right) H_{1}\right) d \sigma_{2} d \sigma_{1}$.

Now, using the assumption that

$$
\int_{0}^{\tau} \int_{0}^{\sigma_{1}} E\left(f\left(\sigma_{1}\right) f\left(\sigma_{2}\right)\right) d \sigma_{1} d \sigma_{2}=\int_{0}^{\tau} \int_{0}^{\sigma_{1}} \delta\left(\sigma_{1}-\sigma_{2}\right) d \sigma_{2} d \sigma_{1}=\tau
$$

we obtain that if we let $\tilde{\rho}=E(\rho(t))$,

$$
\tilde{\rho}(\tau)=\tilde{\rho}(0)-i\left[H_{0}, \tilde{\rho}\right] \tau+\left[i H_{1},\left[i H_{1}, \tilde{\rho}\right]\right] \tau+O\left(\tau^{2}\right),
$$

where, we have only retained terms of the order $\tau$. This, then gives us that

$$
\frac{d \tilde{\rho}}{d \tau}=-i\left[H_{0}, \tilde{\rho}\right]+L(\tilde{\rho}),
$$

where $L(\tilde{\rho})=k\left[i H_{1},\left[i H_{1}, \tilde{\rho}\right]\right]$. For notational simplicity, we write $\tilde{\rho}$ as $\rho$.

The evolution of the density operator for the open quantum system is no longer isospectral. The effect of the term $L(\rho)$ is then to reduce the value of $\operatorname{tr}\left(\rho^{2}\right)$. For instance,

$$
\frac{d \operatorname{tr}\left(\rho^{2}(t)\right)}{d t}=\operatorname{tr}\left(\left[H_{1}, \rho\right]^{2}\right),
$$

where $\operatorname{tr}\left(\left[H_{1}, \rho\right]^{2}\right) \leq 0$, implying that $\operatorname{tr}\left(\rho^{2}\right)$ decreases with time. The effect of the coupling to an external heat bath is to transform a pure state into a mixed state.

A more general form of $L(\rho)$ is

$$
L(\rho)=\sum_{j} k_{j}\left[H_{j},\left[H_{j}, \rho\right]\right]
$$

arising because of random modulations of Hamiltonians $H_{j}$ with fluctuations that are uncorrelated. There are many interesting problems involving control of open quantum systems in the presence of dissipation. The operator $L(\rho)$ is a negative definite operator, such that $\frac{d \operatorname{Tr}\left(\rho^{2}\right)}{d t}=\operatorname{Tr}(\rho L(\rho)) \leq 0$. If we measure the entropy of the ensemble of quantum systems by

$$
S=1-\operatorname{tr}\left(\rho^{2}\right),
$$


also termed Renyi entropy, then observe that pure states have entropy 0 and the effect of the decoherence is to increase the entropy of the system.

In NMR experiments, fluctuations $f(t)$ in the equation (26) arise because the magnetic field $B$, seen by the spins fluctuates with time due to coupling of the spin ensemble with an external bath. We will study source of these fluctuations subsequently. The equation for the density matrix of the $2 \times 2$ spin system then takes the form

$$
\frac{d \rho}{d t}=-i\left[\omega_{0} \sigma_{z}+f(t) \sigma_{z}+u \sigma_{x}+v \sigma_{y}, \rho\right] .
$$

The resulting master equation then takes the form

$$
\frac{d \rho}{d t}=-i\left[\omega_{0} \sigma_{z}+u \sigma_{x}+v \sigma_{y}, \rho\right]-k\left[\sigma_{z},\left[\sigma_{z}, \rho\right]\right]
$$

When the above equation is written as a Bloch equation, the evolution of the Bloch equation takes the form

$$
\frac{d}{d t}\left[\begin{array}{l}
m_{x} \\
m_{y} \\
m_{z}
\end{array}\right]=\left[\begin{array}{ccc}
-k & -\omega_{0} & v(t) \\
\omega_{0} & -k & -u(t) \\
-v(t) & u(t) & 0
\end{array}\right]\left[\begin{array}{l}
m_{x} \\
m_{y} \\
m_{z}
\end{array}\right]
$$

The constant $k$ is called the transverse relaxation rate and is responsible for the decay of the FID signal with time. Eq. (35) is however not a complete description, because eventually $M$ returns back to the original state $(0,0,1)^{\prime}$. A more general model for the Lindblad equations is [14]

$$
L(\rho)=\sum_{k}\left[A_{k} \rho A_{k}^{\dagger}-\frac{1}{2}\left\{A_{k}^{\dagger} A_{k}, \rho\right\}\right]
$$

where $\{A, B\}=A B+B A$ is the anticommutator. If $A_{k}$ are Hermitian operators, then $L(\rho)$ reduces to the familiar form $\sum_{k}\left[A_{k}\left[A_{k}, \rho\right]\right]$. However, $A_{k}$ in general can have both Hermitian and non-Hermitian parts. If we take

$$
A_{1}=\kappa_{1}\left[\begin{array}{ll}
0 & 0 \\
1 & 0
\end{array}\right] ; \quad A_{2}=\kappa_{2}\left[\begin{array}{ll}
0 & 1 \\
0 & 0
\end{array}\right] ; \quad A_{3}=\kappa_{3}\left[\begin{array}{cc}
1 & 0 \\
0 & -1
\end{array}\right] \text {, }
$$

such that $\kappa_{1}>\kappa_{2}$, then the system in (38) will follow the equation

$$
\frac{d}{d t}\left[\begin{array}{l}
m_{x} \\
m_{y} \\
m_{z}
\end{array}\right]=\left[\begin{array}{ccc}
-k & -\omega_{0} & v(t) \\
\omega_{0} & -k & -u(t) \\
-v(t) & u(t) & -\left(T_{1}\right)^{-1}
\end{array}\right]\left[\begin{array}{c}
m_{x} \\
m_{y} \\
m_{z}
\end{array}\right]+\left[\begin{array}{c}
0 \\
0 \\
m_{0}
\end{array}\right] .
$$

Here $k$ and $T_{1}$ in the above equation depends on $\kappa_{1}, \kappa_{2}$ and $\kappa_{3}$ as in Eq. (38). Then, $m_{z}$ eventually returns to $0 \leq m_{0} \leq 1$, at a characteristic time $T_{1}$, also called the longitudinal relaxation rate. 
Although, the effect of the Lindblad operator $L(\rho)$ in Eq. (33) is to increase the entropy of the system, the most general form of the Lindblad equations as in Eq. (37) can lead to decrease in the entropy of the quantum system when mixed suitably with external control. An important application of this feature is in the field of laser cooling [15], where an interplay between unitary control and Lindblad terms as in Eq. (37) is used to decrease the entropy of the quantum system. We study a concrete example to understand the basic ideas in this subject [16].

2.2. Laser Cooling. Consider again the three-level $\Lambda$ system as depicted in Fig. 1B. The evolution of the density matrix of the three-level $\Lambda$ system is given by

$$
\dot{\rho}=-i[H(t), \rho]+\gamma_{1}\left(E_{1} \rho E_{1}^{\dagger}-\frac{1}{2}\left\{E_{1}^{\dagger} E_{1}, \rho\right\}\right)+\gamma_{2}\left(E_{2} \rho E_{2}^{\dagger}-\frac{1}{2}\left\{E_{2}^{\dagger} E_{2}, \rho\right\}\right),
$$

where $E_{1}=|1\rangle\langle 2|$ and $E_{2}=|3\rangle\langle 2|$.

If the density matrix is diagonal, say

$$
\rho=\left[\begin{array}{ccc}
\lambda_{1} & 0 & 0 \\
0 & \lambda_{2} & 0 \\
0 & 0 & \lambda_{3}
\end{array}\right],
$$

with $\lambda_{1}, \lambda_{2}$ and $\lambda_{3}$ describe the population distribution in three energy states. Then, in the absence of any external controls, the density matrix stays diagonal and the diagonal entries evolve as

$$
\frac{d}{d t}\left[\begin{array}{l}
\lambda_{1} \\
\lambda_{2} \\
\lambda_{3}
\end{array}\right]=\underbrace{\left[\begin{array}{ccc}
0 & \gamma_{1} & 0 \\
0 & -\left(\gamma_{1}+\gamma_{2}\right) & 0 \\
0 & \gamma_{2} & 0
\end{array}\right]}_{A}\left[\begin{array}{c}
\lambda_{1} \\
\lambda_{2} \\
\lambda_{3}
\end{array}\right]
$$

We assume that the system is completely controllable and any unitary rotation $U(t)$ on the three level system in (25) can be synthesized in arbitrary small time. In particular, consider the unitary transformation

$$
P=\left[\begin{array}{lll}
0 & 0 & 0 \\
0 & 0 & 1 \\
0 & 1 & 0
\end{array}\right],
$$

which swaps the population states 2 and 3 . The effect of this unitary transformation on the diagonal of the density matrix is

$$
P\left[\begin{array}{l}
\lambda_{1} \\
\lambda_{2} \\
\lambda_{3}
\end{array}\right]=\left[\begin{array}{c}
\lambda_{1} \\
\lambda_{3} \\
\lambda_{2}
\end{array}\right] .
$$


Then, consider the following sequence of operations,

$$
\exp \left(A t_{n}\right) P \exp \left(A t_{n-1}\right) \ldots P \exp \left(A t_{1}\right)
$$

where $t_{i}$ are chosen long enough so that as $\lambda_{2}$ in Eq. (40) decays below the value $\lambda_{3}$. These operations keep the density matrix diagonal and

$$
\exp \left(A t_{n}\right) P \exp \left(A t_{n-1}\right) \ldots P \exp \left(A t_{1}\right)\left[\begin{array}{l}
\lambda_{1} \\
\lambda_{2} \\
\lambda_{3}
\end{array}\right] \sim\left[\begin{array}{l}
1 \\
0 \\
0
\end{array}\right]
$$

It is clear that the population in state 1 keeps building up, while the population from the state 2 and 3 is eventually drained off. The cooling strategy consists of a sequence of dissipative evolutions and unitary control (with electromagnetic fields) to synthesize the Hamiltonians $H_{23}$ in the Fig. 1B. Therefore by an interplay of external control and evolution of natural dynamics, all the population is eventually driven to state 1 , although one starts with a state where the population is distributed in all the states.

3. Control of Ensembles. We now return to the Bloch equations in (18). As discussed earlier, in NMR experiments, there is dispersion in the parameter $\omega$, as the chemical shifts $\sigma$ of the nuclear spins are dispersed over a certain range. In practice, there is another source of the dispersion. The applied radio-frequency field is not uniform on the whole sample but is dispersed over a range, captured by the parameter $\epsilon \in[1-\delta, 1+\delta]$. The Eq. (18) is then modified to

$$
\dot{X}=\left(\omega \Omega_{z}+\epsilon u_{1}(t) \Omega_{x}+\epsilon v_{1}(t) \Omega_{y}\right) X
$$

Dispersion in the parameters in the system dynamics poses some interesting questions in controllability and control design. Eq. (42) represents a continuum of systems parametrized by $\omega$ and $\epsilon$. Fig. 2 shows that application of the control input $\left(u_{1}(t), v_{1}(t)\right)$ as in Eq. (18), results in poor transfer to the transverse plane for spins with $\omega$ comparable or greater than $A_{\max }$, where $\sqrt{u_{1}^{2}(t)+v_{1}^{2}(t)} \leq A_{\max }$ (The net rotation is around a tilted axis $B_{r}$ as shown in the picture). The control challenge is to steer the ensemble of inhomogeneous systems, to a desired target state, inspite of variation in their internal dynamics, by application of the same control law $\left(u_{1}(t), v_{1}(t)\right)$. We say that the system in Eq. (42) is ensemble controllable if the system can be steered from an initial state of the ensemble described by vector valued function $X_{0}(\omega, \epsilon)$ arbitrarily close to the target state $X_{F}(\omega, \epsilon)$ ( where the distance $\left\|X_{F}(\omega, \epsilon)-X_{0}(\omega, \epsilon)\right\|$ is measured by say an $L_{2}$ distance $\iint\left|X_{F}(\omega, \epsilon)-X_{0}(\omega, \epsilon)\right|^{2} d \omega d \epsilon$ between functions). 
This problem represents a typical problem in the control of quantum systems, with dispersion or uncertainty in the parameters governing the dynamics by using the same control field. The problem of designing excitations, that can steer an ensemble and be robust and immune to dispersion in the dynamics of the spin system is a well studied subject in NMR spectroscopy and extensive literature exists on the subject of so called composite pulses that can correct for dispersion in system dynamics [17]. In many cases of practical interest, one wants to find control inputs that prepare the final state as some desired function of the parameter. For example, slice selective excitation and inversion pulses in magnetic resonance imaging [18, 20, 21]. Only Recently, these problems have been understood and posed as questions in controllability of infinite dimensional systems $[22,23,24]$. A principled study of the controllability of these ensemble of systems reveals aspects of system dynamics, which makes it possible to engineer excitations that can steer a quantum ensemble and be robust to the dispersion in the system dynamics. These problems therefore motivate development of new methods and techniques for studying controllability and constructive controllability of a class of infinite dimensional nonlinear systems.

To fix ideas, we first set the dispersion $\omega$ in Eq. (42) to zero, and only consider dispersion arising due to an inhomogeneous rf-field on the sample, measured by the parameter $\epsilon$. Rewriting Eq. (42), we obtain

$$
\dot{X}=\left(\epsilon u_{1}(t) \Omega_{x}+\epsilon v_{1}(t) \Omega_{y}\right) X .
$$

We now summarize the basic ideas [22], that make it possible to engineer input excitations that can steer the whole ensemble uniformly and be immune to the dispersion in the value of $\epsilon$. Observe for small $d t$, the evolution $U_{1}(\sqrt{d t})=$

$$
\exp \left(-\epsilon \Omega_{y} \sqrt{d t}\right) \exp \left(-\epsilon \Omega_{x} \sqrt{d t}\right) \exp \left(\epsilon \Omega_{y} \sqrt{d t}\right) \exp \left(\epsilon \Omega_{x} \sqrt{d t}\right)
$$

to leading order in $\epsilon$ is $I+\epsilon^{2}\left[\Omega_{x}, \Omega_{y}\right]$, i.e., we can synthesize the generator $\left[\epsilon \Omega_{x}, \epsilon \Omega_{y}\right]=$ $\epsilon^{2} \Omega_{z}$, by back and forth maneuver in the directly accessible directions $\Omega_{x}$ and $\Omega_{y}$. Similarly, the leading order term in the evolution

$$
U_{2}=U_{1}(-\sqrt{d t}) \exp \left(-\epsilon \Omega_{y} d t\right) U_{1}(\sqrt{d t}) \exp \left(\epsilon \Omega_{y} d t\right) .
$$

is $\left[\epsilon \Omega_{y},\left[\epsilon \Omega_{x}, \epsilon \Omega y\right]\right]=\epsilon^{3} \Omega_{x}$. By successive Lie brackets, terms of the type $\epsilon^{2 k+1} \Omega_{x}$ can be synthesized to leading order. Now using $\left\{\epsilon \Omega_{x}, \epsilon^{3} \Omega_{x}, \ldots, \epsilon^{2 n+1} \Omega_{x}\right\}$ as generators, we can produce an evolution

$$
\exp \left\{\sum_{k=0}^{n} c_{k} \epsilon^{2 k+1} \Omega_{x}\right\},
$$


where $n$, and the coefficients $c_{k}$ can be so chosen so that

$$
\sum_{k=0}^{n} c_{k} \epsilon^{2 k+1} \approx \theta
$$

for all $\epsilon \in[1-\delta, 1+\delta]$. Therefore, an evolution $\exp \left(\theta \Omega_{x}\right)$ can be synthesized for all values of $\epsilon$ to any desired accuracy. Therefore, one achieves robustness with dispersion to $\epsilon$ by generating effective generators with arbitrary high powers of the dispersion parameter $\epsilon$.

Contrast the situation in Eq. (43), with the following control system, the well studied non-holonomic integrator,

$$
\frac{d}{d t}\left[\begin{array}{l}
x \\
y \\
z
\end{array}\right]=\epsilon u\left[\begin{array}{c}
1 \\
0 \\
-y
\end{array}\right]+\epsilon v\left[\begin{array}{l}
0 \\
1 \\
x
\end{array}\right] .
$$

If $\epsilon$ is fixed, then the system in Eq. (45) is controllable as the vector fields

$$
f=\left[\begin{array}{c}
1 \\
0 \\
-y
\end{array}\right] ; g=\left[\begin{array}{l}
0 \\
1 \\
x
\end{array}\right]
$$

generate the vector field $[f, g]=[0,0,1]^{\prime}$. The three vector fields $f, g,[f, g]$, then span the three dimensional space. However, the Lie algebra generated by $f, g$ is nilpotent and therefore $[\cdot,[f, g]]=0$. The dispersion parameter $\epsilon$, cannot be raised to higher powers by iterated brackets and therefore such an ensemble of inhomogeneous nilpotent systems is not ensemble controllable. On the contrary, the Lie algebra $\mathfrak{g}=s o(3)$ generated by $\Omega_{x}$ and $\Omega_{y}$ in (43) is semi-simple (implying $[\mathfrak{g}, \mathfrak{g}]=\mathfrak{g}$ ) and therefore the iterated Lie brackets never terminate, allowing for design of robust input excitations. Similarly, Linear systems

$$
\frac{d X}{d t}=A X+\epsilon B u
$$

cannot be steered uniformly by application of the same control input $u(t)$, as the output, for $X(0)=0$ is

$$
X(t)=\epsilon \int_{0}^{t} \exp (A(t-\tau)) B(\tau) u(\tau) d \tau
$$

which is just a linear function of the input. No matter how $u(\tau)$ is modulated, the output depends linearly on the input.

Interesting control design problems arise in the manipulation of inhomogeneous quantum ensembles. To provide a flavor for such problems, we describe one synthesis 
method [25] for designing input excitations, that robustly steer the inhomogeneous ensemble in Eq. (43). This synthesis method has recently been used in the design of rf pulse sequences in NMR spectroscopy that are robust to rf-inhomogeneity. Consider the following rotations obtained by alternate rotations around $x$ and $y$ axis for appropriate duration. Let

$$
\begin{aligned}
& U_{1}=\exp \left(k \pi \Omega_{x} \epsilon\right) \exp \left(\frac{\beta_{k}}{2} \Omega_{y} \epsilon\right) \exp \left(-k \pi \Omega_{x} \epsilon\right), \\
& U_{2}=\exp \left(-k \pi \Omega_{x} \epsilon\right) \exp \left(\frac{\beta_{k}}{2} \Omega_{y} \epsilon\right) \exp \left(k \pi \Omega_{x} \epsilon\right) .
\end{aligned}
$$

Now, by choosing $\beta_{k}$, small enough, we have

$$
V_{k}=U_{2} U_{1} \sim \exp \left(\epsilon \beta_{k} \Omega_{y} \cos (k \pi \epsilon)\right) .
$$

Then, a sequence of transformations

$$
\Pi_{k}\left(V_{k}\right)^{n_{k}} \sim \exp \left(\epsilon \sum_{k} \alpha_{k} \cos (k \pi \epsilon) \Omega_{y}\right),
$$

where $\alpha_{k}=n \beta_{k}$. Now, the coefficients $\alpha_{k}$ can be so chosen that

$$
\sum_{k} \alpha_{k} \cos (k \pi \epsilon)=\frac{\theta}{\epsilon}
$$

for $1-\delta \leq \epsilon \leq 1$, with $0<\delta<1$.

Therefore,

$$
\Pi_{k}\left(V_{k}\right)^{n_{k}} \sim \exp (\theta)
$$

The actual control input to Eq. (15) consists of oscillatory input $(u, v)=(A \cos ($ $\left.\left.\omega_{0} t+\phi\right), 0\right)$, where the phase $\phi$ is switched between $0, \frac{\pi}{2}$ and $\pi$ to achieve rotations around $x, y$ and $-x$ axis respectively. Fig. 5 shows this control input and its pictorial depiction as a pulse sequence.

\subsection{Controllability of Bloch Equations in the Presence of Frequency}

Dispersion. Now, consider the Bloch equations as in Eq.(18), with dispersion in the Larmor frequencies. We now like to show that this system is ensemble controllable with respect to the dispersion in the parameter $\omega$.

There is an important conceptual issue that emerges in controllability analysis of such problems. In studying controllability of control systems of the kind

$$
\dot{x}=(A+u B) x,
$$




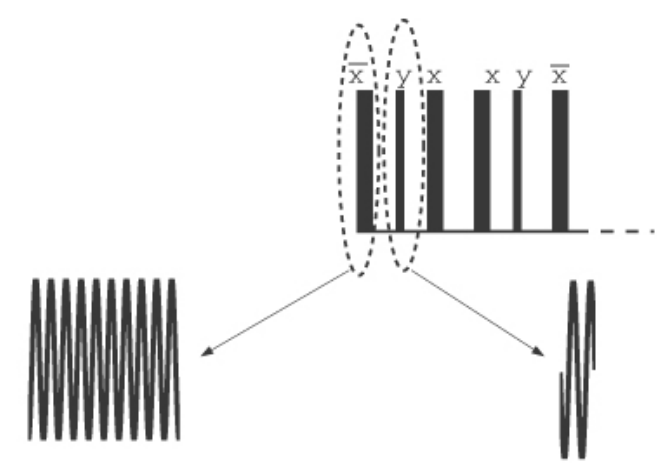

FIG. 5. The above figure shows the sequence of pulses with alternate phases as described in Eq. (47), which forms the building block of a composite pulse train as in Eq. (49). Each pulse is an oscillatory control input the phase of which is changed from pulse to pulse.

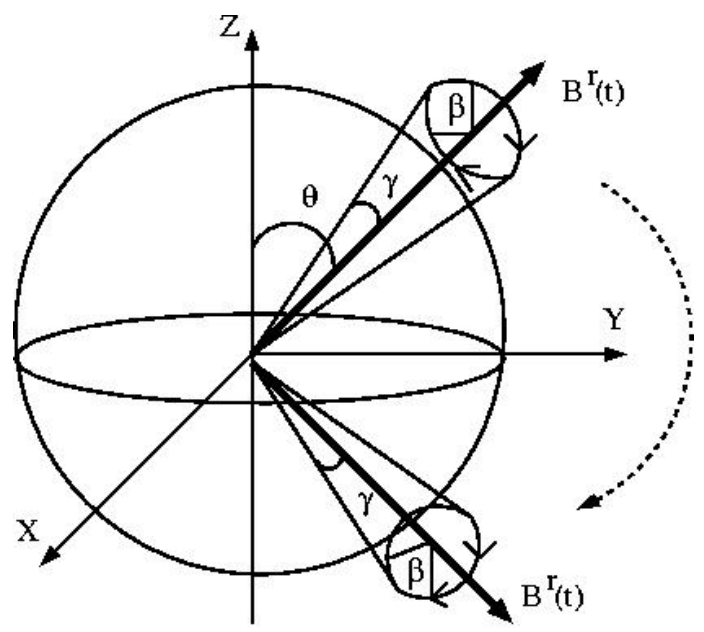

FIG. 6. The figure shows how the vector $X$ in equation $E q$. (15) can be dragged from $(x, y, z)=$ $(0,0,1)$ to $(0,0,-1)$, independent of value of $\omega$ by slowly varying $\tilde{\omega}(t)$ in Eq. (54).

evolving on compact Lie groups, it is possible to synthesize commutators of the kind $[A, B]$ to leading order, by an evolution

$$
\exp (-A d t) \exp (-B d t) \exp (A d t) \exp (B d t)
$$

where the backward evolution $\exp (-A d t)$ is generated by letting the forward map $\exp (A t)$ evolve for sufficient period of time. The free evolution on a compact group almost returns back after sufficient time. However, the situation is different for a continuum of such systems as in Eq. (18). In the presence of continuum of frequencies $\omega \in[-B, B]$, given small time $d t$, there is no forward evolution time $T$, such that $\exp (\omega T)=\exp (-\omega d t)$, for all $\omega \in[-B, B]$. However, by using control, we can synthesize an effective backward evolution. Two limits are of particular interest here. 
We first assume that our control inputs in Eq. (18) are unbounded apriori $\left(A_{\max } \gg \omega\right)$. Note, because of the assumption of strong fields, we can reverse the evolution of the drift term in Eq. (18),

$$
\exp \left(\pi \Omega_{x}\right) \exp \left(\omega \Omega_{z} d t\right) \exp \left(-\pi \Omega_{x}\right)=\exp \left(-\omega \Omega_{z} d t\right)
$$

where $\omega \in[-B, B]$, and the $\pi$ rotations like $\exp \left(\pi \Omega_{x}\right)$ can be produced in negligible time. Now, we consider the case when the controls $u$ and $v$ are bounded, i.e., $\sqrt{u^{2}(t)+v^{2}(t)} \leq A_{\max }$ for all $t$, so that we cannot produce rotations of the type $\exp \left(-\Omega_{x} \pi\right)$ in an arbitrarily small time as in Eq. (52).

Nonetheless, the system is ensemble controllable as will be shown below. The key to showing this is to produce the backward evolution of the drift term, $\exp \left(-\omega \Omega_{z} d t\right)$. This helps us to generate higher-order Lie brackets with the drift term containing higher powers of dispersion parameters $\omega$, which can be combined to produce an evolution that is robust to $\omega$. Our construction initially employs the well known construction in physics literature called the adiabatic following, which helps to synthesize an evolution $\exp \left(-\omega \Omega_{z} d t\right)$. This construction can be used to show ensemble controllability with respect to both Larmor dispersion and rf inhomogeneity in the Bloch equations (43). Adiabatic following is a technique, widely employed in variety of experiments involving control of quantum systems as it is robust to inhomogeneity in the system dynamics. It is of independent interest from the perspective of non-linear control.

3.1.1. Adiabatic Following. Consider the Bloch equations with only Larmor dispersion as in Eq. (42), which we rewrite to reflect ensemble of systems with $\omega$ dependence.

$$
\dot{X}(t, \omega)=\left[\omega \Omega_{z}+u \Omega_{y}+v \Omega_{x}\right] X(t, \omega),
$$

where $\omega \in[-B, B]$. Let $\left(u_{1}(t), v_{1}(t)\right)=A(t)(\cos \phi(t), \sin \phi(t)), A=\sqrt{u_{1}^{2}(t)+v_{1}^{2}(t)}$. We then slowly vary $\dot{\phi}(t)$ from an initial value $\dot{\phi}(0) \ll-B$ to $\dot{\phi} \gg B$. We show that if the change in $\dot{\phi}(t)$ is slow enough, all systems as in $(53)$ can be steered from $(0,0,1)^{T}$ to $(0,0,-1)^{T}$. We first make a change of coordinates

$$
Y(t, \omega)=\exp \left[-\phi(t) \Omega_{z}\right] X(t, \omega)
$$

The resulting system then takes the form

$$
\dot{Y}(t, \omega)=\left([\omega-\tilde{\omega}(t)] \Omega_{z}+A \Omega_{y}\right) Y(t, \omega),
$$

where $\tilde{\omega}(t)=\dot{\phi}(t)$. Thus, the effective generator of motion is

$$
(\omega-\tilde{\omega}(t)) \Omega_{z}+A \Omega_{y} .
$$


In the standard physics terminology, the Bloch vector $Y(t, \omega)$ rotates around the effective field $B^{r}=A \mathbf{j}+[\omega-\tilde{\omega}(t)] \mathbf{k}$ (see Fig. 6) and has the net magnitude of rotation

$$
\left|B^{r}\right|=\sqrt{(A)^{2}+[\omega-\tilde{\omega}(t)]^{2}}=A \sqrt{1+\tan ^{2} \theta} .
$$

The angle $\theta$ through which $B^{r}$ is tilted with respect to $A$ is defined by

$$
\tan \theta=\frac{\omega-\tilde{\omega}(t)}{A} .
$$

By differentiating (54), we get the rate of change for the angle $\theta(t)$

$$
\dot{\theta}=\frac{-\dot{\tilde{\omega}}(t)}{A} \cos ^{2} \theta
$$

The maximum value of the RHS of the above expression is obtained when $\theta=0$ and we have

$$
|\dot{\theta}|_{\max }=\frac{|\dot{\tilde{\omega}}(t)|}{A} .
$$

In addition, the smallest rate of rotation of $X$ around $B^{r}$ is $A$. This happens when $\tilde{\omega}(t)=\omega$, i.e. $\theta$ in $(54)$ is 0 . If we vary $\tilde{\omega}(t)$ slowly enough so that $\left|\dot{\theta}_{\max }\right| \ll A$, i.e.,

$$
|\dot{\tilde{\omega}}(t)| \ll A^{2}
$$

from $\theta(0)=\frac{\pi}{2}$ to the final state $-\frac{\pi}{2}$ such that the variation is slow, then $X(t, \omega)$ for all $\omega$ follows the effective field (remains locked around $B^{r}$ ) from $(0,0,1)^{T}$ to $(0,0,-1)^{T}$ simultaneously. This can be seen by the following averaging argument. Observe that in Fig. 6, the rate of change of the angle $\gamma$ at time $t$ is a function of $\dot{\theta}$ and $\beta$, i.e.,

$$
\frac{d \gamma}{d t}=h(\dot{\theta}, \beta)
$$

where the angles $\gamma$ and $\beta$ are defined in Fig. 6 . Because $\beta$ changes at a much faster rate compared to $\theta$, i.e., $\dot{\beta} \gg \dot{\theta}$, the time scale separation gives

$$
\gamma(t+\tau)-\gamma(t)=\int_{0}^{\tau} h(\dot{\theta}(t), \beta(t+\sigma)) d \sigma \approx 0,
$$

where $\tau$ is the period for $\beta$ to rotate by $2 \pi$ over which $\dot{\theta}(t)$ is supposed to be constant. Therefore, we can maintain $\gamma(t)$ very small throughout, i.e., $0 \leq \gamma(t) \leq \varepsilon$ for all $t$, and $\varepsilon$ can be controlled by the rate $\dot{\theta}(t)$. Now note that $\theta(T) \approx \pi$ and hence $X(T, \omega) \approx-X_{0}$ for $\omega \in[-B, B]$, where $X_{0}=(0,0,1)^{T}$. As a result, there exists a net evolution $U(\omega)$ for all $\omega \in[-B, B]$ such that

$$
U(\omega) X_{0} \approx-X_{0}
$$


Therefore, by doing an Euler angle decomposition, we can decompose

$$
U(\omega)=\exp \left(f(\omega) \Omega_{z}\right) \exp \left(\pi \Omega_{x}\right) \exp \left(g(\omega) \Omega_{z}\right)
$$

where $f(\omega)$ and $g(\omega)$ are some functions of $\omega$. Then, observe,

$$
U^{2}(\omega) \sim \exp \left(f(\omega) \Omega_{z}\right) \exp \left(-g(\omega) \Omega_{z}\right) \exp \left(-f(\omega) \Omega_{z}\right) \exp \left(g(\omega) \Omega_{z}\right)=\mathbf{1} .
$$

$$
U(\omega) \exp \left(\omega \Omega_{z} t\right) U(\omega)=\exp \left(-\omega \Omega_{z} t\right)
$$

The propagator $U(\omega)$ can be used to reverse the direction of drift in (56). Now, constructions as described before can be used to produce any rotation in Eq. (42) as a function of $\omega$. This approximation in Eq. (55) is in $L_{2}$ sense as described earlier and can be made arbitrarily good by regulating how slowly $\dot{\theta}$ is changed. In fact, it is possible to write down the explicit time-dependent control law that will transfer $X_{0}$ to $-X_{0}$. This is the well studied complex hyperbolic secant pulse [18] and is very interesting from the perspective of nonlinear control.

We have sketched the basic ideas required to show that Bloch Eq. (18) can be steered to a target state that has desired dependence of the drift parameter $\omega$. In many applications in NMR and magnetic resonance imaging (MRI), one requires input control design that only excites spins with specific value of $\omega$ to the equator with a final state that depends in a specified way of $\omega$ and leaves other spins invariant.

3.2. Shinnar Roux Algorithm and Nonlinear Control Design. We now present a well used $[20,21]$ design strategy for generating input excitations that steers the system in Eq. (18) from an initial $X(0)=(0,0,1)^{\prime}$ to a target state on the transverse plane say $\left((X(\omega), Y(\omega), 0)^{\prime}\right.$, for $\omega \in\left[-B_{1}, B_{1}\right]$, where $X(\omega)$ and $Y(\omega)$ can be specified. This design strategy is intriguing as it steers an whole ensemble of nonlinear systems in to a desired final target point. This expository subsection is aimed at motivating further method development in nonlinear control design to address problems of the kind presented in this section.

The solution to the Bloch equation (14) is a rotation $X(T)=R X(0)$, where $R \in S O(3)$. We work with $S U(2)$ representation of these rotations, where a rotation by angle $\phi$ around the unit vector $\left(n_{x}, n_{y}, n_{z}\right)$ has a representation of the form $U=$ $\left[\begin{array}{cc}\alpha & -\beta^{*} \\ \beta & \alpha^{*}\end{array}\right]$, where $\alpha$ and $\beta$ are the Cayley-Klein parameters satisfying $\alpha=\cos \frac{\phi}{2}-$ $i n_{z} \sin \frac{\phi}{2}, \beta=-i\left(n_{x}+i n_{y}\right) \sin \frac{\phi}{2}$, and $\alpha \alpha^{*}+\beta \beta^{*}=1$. The Bloch equation then takes the form

$$
\dot{U}=-\frac{i}{2}\left[\begin{array}{cc}
\omega & u-i v \\
u+i v & -\omega
\end{array}\right] U
$$


The rotation $U$ is simply represented by its first column (also termed spinor representation) $\psi=\left[\begin{array}{l}\alpha \\ \beta\end{array}\right]$. We first consider piecewise-constant controls $u(t)$ and $v(t)$. The net rotation under these controls can be represented as successive rotations $U=U_{n} U_{n-1} \ldots U_{1} U_{0}$, where $U_{j}=\left[\begin{array}{cc}a_{j} & -b_{j}^{*} \\ b_{j} & a_{j}^{*}\end{array}\right]$ and $a_{j}, b_{j}$ are the Cayley-Klein parameters for the $j$ th interval. Defining the multiplication of the matrices $U_{j}$ up to $k$ by

$$
\left[\begin{array}{cc}
\alpha_{k} & -\beta_{k}^{*} \\
\beta_{k} & \alpha_{k}^{*}
\end{array}\right]=\left[\begin{array}{cc}
a_{k} & -b_{k}^{*} \\
b_{k} & a_{k}^{*}
\end{array}\right] \cdots\left[\begin{array}{cc}
a_{0} & -b_{0}^{*} \\
b_{0} & a_{0}^{*}
\end{array}\right],
$$

the effect of the controls can then be calculated by propagating the spinor

$$
\left[\begin{array}{c}
\alpha_{k} \\
\beta_{k}
\end{array}\right]=\left[\begin{array}{cc}
a_{k} & -b_{k}^{*} \\
b_{k} & a_{k}^{*}
\end{array}\right]\left[\begin{array}{c}
\alpha_{k-1} \\
\beta_{k-1}
\end{array}\right]
$$

with the initial condition $\left[\begin{array}{l}\alpha_{0} \\ \beta_{0}\end{array}\right]=\left[\begin{array}{l}1 \\ 0\end{array}\right]$. The duration $\Delta t$, over which the controls $u$ and $v$ are constant can be chosen small enough such that, the net rotation can be decomposed into two sequential rotations since

$$
e^{\left(\omega \Omega_{z}+u \Omega_{y}-v \Omega_{x}\right) \Delta t} \approx e^{\left(u \Omega_{y}-v \Omega_{x}\right) \Delta t} e^{\omega \Omega_{z} \Delta t} .
$$

Under this assumption, we can write the rotation $U_{k}$ as a rotation around $z$-axis by an angle $\omega \Delta t$ followed by a rotation about the applied control fields by an angle $\phi_{k}$ in the $S U(2)$ representation

$$
U_{k}=\left[\begin{array}{cc}
C_{k} & -S_{k}^{*} \\
S_{k} & C_{k}
\end{array}\right]\left[\begin{array}{cc}
z^{1 / 2} & 0 \\
0 & z^{-1 / 2}
\end{array}\right]
$$

where

$$
\begin{array}{lc}
C_{k}=\cos \frac{\phi_{k}}{2}, & S_{k}=-i e^{i \theta_{k}} \sin \frac{\phi_{k}}{2}, \\
\phi_{k}=A_{k} \Delta t, & \theta_{k}=\tan ^{-1} \frac{v_{k}}{u_{k}}, \\
A_{k}=\sqrt{u_{k}^{2}+v_{k}^{2}}, & z=e^{-i \omega \Delta t} .
\end{array}
$$

Plugging (58) into (57), we get the recursion relation of the spinor 


$$
\left[\begin{array}{c}
\alpha_{k} \\
\beta_{k}
\end{array}\right]=z^{\frac{1}{2}}\left[\begin{array}{cc}
C_{k} & -S_{k}^{*} z^{-1} \\
S_{k} & C_{k} z^{-1}
\end{array}\right]\left[\begin{array}{c}
\alpha_{k-1} \\
\beta_{k-1}
\end{array}\right] .
$$

Defining $P_{k}=z^{-k / 2} \alpha_{k}$ and $Q_{k}=z^{-k / 2} \beta_{k}$, the recursion may then be reduced to

$$
\left[\begin{array}{c}
P_{k} \\
Q_{k}
\end{array}\right]=\left[\begin{array}{cc}
C_{k} & -S_{k}^{*} z^{-1} \\
S_{k} & C_{k} z^{-1}
\end{array}\right]\left[\begin{array}{c}
P_{k-1} \\
Q_{k-1}
\end{array}\right]
$$

with the initial condition

$$
\left[\begin{array}{l}
P_{0} \\
Q_{0}
\end{array}\right]=\left[\begin{array}{l}
1 \\
0
\end{array}\right]
$$

From the recursion (60) and the initial condition (61), the spinor at the $n$th time step can be represented as the $(n-1)$-order polynomials in $z, P_{n}(z)=\sum_{k=0}^{n-1} p_{k} z^{-k}$ and $Q_{n}(z)=\sum_{k=0}^{n-1} q_{k} z^{-k}$ where $\left|P_{n}(z)\right|^{2}+\left|Q_{n}(z)\right|^{2}=1$. The parameter $z$ encodes the dispersion parameter $\omega$. The desired final states of an ensemble of systems in (14), described by Cayley-Klein parameters, are two functions of $z$, and hence of $\omega$. We can now design two polynomials $P_{n}(z)$ and $Q_{n}(z)$ such that we can approximate any desired smooth functions $F_{\alpha}(z)$ and $F_{\beta}(z)$ satisfying $\left|F_{\alpha}(z)\right|^{2}+\left|F_{\beta}(z)\right|^{2}=1$, which characterizes the desired spinor we want as function of $z$. Now, we can work backwards and compute the $u_{k}^{\prime} s$ and $v_{k}^{\prime} s$ that will produce $P_{n}(z)$ and $Q_{n}(z)$. Note, by multiplying both sides of (60) by the inverse of the rotation matrix we get

$$
\left[\begin{array}{c}
P_{k-1} \\
Q_{k-1}
\end{array}\right]=\left[\begin{array}{c}
C_{k} P_{k}+S_{k}^{*} Q_{k} \\
\left(-S_{k} P_{k}+C_{k} Q_{k}\right) z
\end{array}\right] .
$$

We have a backward recursion where we use the knowledge of coefficients of $P_{k}(z)$ and $Q_{k}(z)$ to compute $P_{k-1}(z)$ and $Q_{k-1}(z)$. This is the well known Shinnar Roux $[20,21]$ algorithm. Because $P_{k-1}(z)$ and $Q_{k-1}(z)$ are lower order polynomials, the leading term in $P_{k-1}$ and the low-order term in $Q_{k-1}(z)$ must drop out

$$
\begin{aligned}
C_{k} P_{k, k-1}+S_{k}^{*} Q_{k, k-1} & =0, \\
-S_{k} P_{k, 0}+C_{k} Q_{k, 0} & =0,
\end{aligned}
$$

where $P_{k, m}$ denotes the coefficient of $z^{-m}$ term in $P_{k}(z)$. These two equations are equivalent. Choosing (64) and combining it with (60), we get $\frac{Q_{k, 0}}{P_{k, 0}}=\frac{-i e^{i \theta_{k}} \sin \frac{\phi_{k}}{2}}{\cos \frac{\phi_{k}}{2}}$. This gives the flip angle $\phi_{k}=2 \tan ^{-1}\left|\frac{Q_{k, 0}}{P_{k, 0}}\right|$, and the phase $\theta_{k}=\measuredangle\left(\frac{i Q_{k, 0}}{P_{k, 0}}\right)$. The controls are then $u_{k}=\frac{\phi_{k}}{\Delta t} \sin \theta_{k}$, and $v_{k}=\frac{\phi_{k}}{\Delta t} \cos \theta_{k}$. These expressions for controls coupled with the inverse recursion in (62) construct the piecewise constant controls $u_{k}, v_{k}$ that 
generate polynomial approximations $P_{n}(z)$ and $Q_{n}(z)$ of the target function $F_{\alpha}(z)$ and $F_{\beta}(z)$.

In particular, if we choose $F_{\beta}(z)=-i \sin \frac{\phi}{2}$ and $F_{\alpha}(z)=\cos \frac{\phi}{2}$, we obtain a broadband rotation (uniform over all $\omega$ ) around $x$ axis by angle $\phi$ and similarly by choosing $F_{\beta}(z)=\sin \frac{\phi}{2}$ and $F_{\alpha}(z)=\cos \frac{\phi}{2}$, we obtain an approximation to a broadband rotation around $y$ axis by angle $\phi$.

There are numerous open problems in quantum control involving design of input excitations that are robust to inhomogeneities in the system dynamics. For sake of brevity, we only mention one here. Finding explicit $\left(u_{1}(t), v_{1}(t)\right)$ in (Eq. 42) that steers the system from an initial $X(0)=(0,0,1)^{\prime}$ to the target state $\left((1,0,0)^{\prime}\right.$ for all values of $\omega$ and $\epsilon$ is an important challenge in NMR and MRI. There are important control design challenges when $\omega(t)$ and $\epsilon(t)$ are both time varying. Recently, numerical methods like gradient ascent algorithms have been used for design of pulse sequences that are robust to dispersions in parameters of the spin system [26, 27, 28]. However more constructive techniques like the Shinnar Roux algorithm are of fundamental interest in design of control inputs to steer the nonlinear control system in (Eq. 42)in a robust way.

4. Coupled Spin Dynamics. Until now, we have described bilinear control systems that arise in the control of spin $\frac{1}{2}$ or an ensemble of spin $\frac{1}{2}$. A rich class of model control problems arise, when one considers dynamics of two coupled spin $\frac{1}{2}$. The dynamics of two coupled spin $\frac{1}{2}$, forms the basis for the field of quantum information processing and computing and is fundamental in multidimensional NMR spectroscopy experiments as detailed subsequently. Let $|0\rangle$ and $|1\rangle$ represent a choice of the orthogonal basis for the Hilbert space of state of the spin $\frac{1}{2}$, for example, the eigenstates of the operator $\sigma_{z}$, with eigenvalues $\frac{1}{2}$ and $-\frac{1}{2}$ respectively. The joint Hilbert space of the coupled spin system is the tensor product of the individual one of these. A possible choice of the basis for the joint Hilbert space is the tensor product of basis for individual of these spaces (also termed the product operator basis) and we represent these basis as $|00\rangle,|01\rangle,|10\rangle$, and $|11\rangle$. Where

$$
|00\rangle=\left[\begin{array}{l}
1 \\
0
\end{array}\right] \otimes\left[\begin{array}{l}
1 \\
0
\end{array}\right]=\left[\begin{array}{l}
1 \\
0 \\
0 \\
0
\end{array}\right]
$$

An arbitrary vector in this space takes the form

$$
a|00\rangle+b|01\rangle+c|10\rangle+d|11\rangle .
$$

Not all the vectors in the joint Hilbert space can be written as the tensor product 
$\left|\phi_{1}\right\rangle \otimes\left|\phi_{2}\right\rangle$. Vectors that can be decomposed in this way are called separable states and those that cannot are termed entangled states. For example, the states

$$
\begin{aligned}
\left|\psi_{A} \pm\right\rangle & =\frac{|00\rangle \pm|11\rangle}{\sqrt{2}} \\
\left|\psi_{B} \pm\right\rangle & =\frac{|01\rangle \pm|10\rangle}{\sqrt{2}}
\end{aligned}
$$

are examples of entangled states and are given special name of the Bell states.

The Hamiltonian for a system of two coupled spins then takes the general form

$$
H_{0}=\sum a_{\mu} \sigma_{\mu} \otimes \mathbf{1}+\sum b_{\nu} \mathbf{1} \otimes \sigma_{\nu}+\sum J_{\mu \nu} \sigma_{\mu} \otimes \sigma_{\nu},
$$

where $\mu, \nu \in\{x, y, z\}$. The Hamiltonians $\sigma_{\mu} \otimes \mathbf{1}$ and $\mathbf{1} \otimes \sigma_{\nu}$ are termed local Hamiltonians and the Hamiltonian

$$
H_{c}=\sum c_{\mu \nu} \sigma_{\mu} \otimes \sigma_{\nu}
$$

the coupling or interaction Hamiltonian. The local Hamiltonians only operate on one of the spins. For example, $\sigma_{\mu} \otimes \mathbf{1}$ only transforms the first spin (label as $I$ )

$$
\sigma_{\mu} \otimes \mathbf{1}\left|\phi_{1}\right\rangle \otimes\left|\phi_{2}\right\rangle=\left(\sigma_{\mu}\left|\phi_{1}\right\rangle\right) \otimes\left|\phi_{2}\right\rangle .
$$

Similarly $1 \otimes \sigma_{\mu}$, only transforms the second spin (label as $S$ ).

The following notation is therefore common place in the NMR literature.

$$
I_{\mu}=\sigma_{\mu} \otimes 1 \quad ; \quad S_{\nu}=1 \otimes \sigma_{\nu} .
$$

The operators $I_{\mu}$ and $S_{\nu}$ commute and therefore $\exp \left(-i \sum_{\mu} a_{\mu} I_{\mu}+\sum_{\nu} b_{\nu} S_{\nu}\right)=$

$$
\exp \left(-i \sum_{\mu} a_{\mu} I_{\mu}\right) \exp \left(-i \sum_{\nu} b_{\nu} S_{\nu}\right)=\left(\exp \left(-i \sum_{\mu} a_{\mu} \sigma_{\mu}\right) \otimes \mathbf{1}\right)\left(\mathbf{1} \otimes \exp \left(-i \sum_{\nu} b_{\nu} \sigma_{\nu}\right),\right.
$$

and therefore

$$
\begin{aligned}
& \exp \left(-i \sum_{\mu} a_{\mu} I_{\mu}+\sum_{\nu} b_{\nu} S_{\nu}\right)\left|\phi_{1}\right\rangle \otimes\left|\phi_{2}\right\rangle \\
= & \left(\exp \left(-i \sum_{\mu} a_{\mu} \sigma_{\mu}\right)\left|\phi_{1}\right\rangle\right) \otimes\left(\exp \left(-i \sum_{\nu} b_{\nu} \sigma_{\nu}\right)\left|\phi_{2}\right\rangle\right),
\end{aligned}
$$

implying that the evolution of local Hamiltonians preserves separable states. The unitary transformations of the kind

$$
\exp \left(-i \sum_{\mu} a_{\mu} \sigma_{\mu}\right) \otimes \exp \left(-i \sum_{\nu} b_{\nu} \sigma_{\nu}\right)
$$

obtained by evolution of local Hamiltonians are called local unitary transformations. 
Entangled states can be generated starting from separable states by letting the coupling Hamiltonian evolve. The coupling Hamiltonian can be written as

$$
H_{c}=\sum J_{\mu \nu} I_{\mu} S_{\nu}
$$

Written explicitly, some of these matrices take the form

$$
I_{z}=\sigma_{z} \otimes \mathbf{1}=\frac{1}{2}\left[\begin{array}{cccc}
1 & 0 & 0 & 0 \\
0 & 1 & 0 & 0 \\
0 & 0 & -1 & 0 \\
0 & 0 & 0 & -1
\end{array}\right] .
$$

and

$$
I_{z} S_{z}=\sigma_{z} \otimes \sigma_{z}=\frac{1}{4}\left[\begin{array}{cccc}
1 & 0 & 0 & 0 \\
0 & -1 & 0 & 0 \\
0 & 0 & -1 & 0 \\
0 & 0 & 0 & 1
\end{array}\right]
$$

The 15 operators,

$$
-i\left\{I_{\alpha}, S_{\beta}, I_{\alpha} S_{\beta}\right\}
$$

for $\alpha, \beta \in\{x, y, z\}$, form the basis for the Lie algebra $\mathfrak{g}=s u(4)$, the $4 \times 4$, skew Hermitian matrices. For the coupled two spins, the generators $-i H_{0},-i H_{i} \in s u(4)$ and the evolution operator $U(t)$ in Eq. (14) is an element of $S U(4)$, the $4 \times 4$, unitary matrices of determinant 1 . The density matrix for a two spin system is then $4 \times 4$ Hermitian matrix with trace 1 and can be written as

$$
\rho=\frac{1}{4}+\sum_{\mu} a_{\mu} I_{\mu}+\sum_{\nu} b_{\nu} S_{\nu}+\sum_{\mu \nu} J_{\mu \nu} I_{\mu} S_{\nu} .
$$

It is customary to omit $\mathbf{1}$ in the formula for (76), as it doesnot transform under a unitary transformation. The various terms in the decomposition of the density matrix have a special meaning. A density matrix $\rho_{I}=\frac{1}{4}+\alpha_{I} I_{z}$, corresponds to the state of the spin ensemble, where there are excess of spins $I$ oriented along the $z$ axis, the $B_{0}$ field direction, while there is no preferred orientation for spin $S$. Similarly, a density matrix $\rho_{S}=\frac{1}{4}+\alpha_{S} S_{z}$, corresponds to the state of the spin ensemble, when there are excess of spins $S$ oriented along the $B_{0}$ field direction, while there is no preferred orientation for spins $I$.

Numerous experiments in NMR spectroscopy involve synthesizing unitary transformations that require interaction between the spins (evolution of the coupling Hamiltonian). These experiments involve transferring, for example the initial state of the spin ensemble represented by a density operator of the kind $\rho_{I}$ to a final density operator of the kind $\rho_{S}$ and involves evolution of interaction Hamiltonians. Such transfer 
experiments are used to improve sensitivity of the measurement and will be discussed subsequently. Similarly, many protocols in quantum communication and information processing [35] involve synthesizing entangled states as in Eq. (66) starting from the separable states. This again requires evolution of interaction Hamiltonians between the spins.

A typical feature of many of these problems is that evolution of interaction Hamiltonians takes significantly longer than the time required to generate local unitary transformations. Local unitary transformations on spins are obtained by application of rf-pulses, whose strength may be orders of magnitude larger than the couplings between the spins. This poses the problem of time optimal control of coupled spin dynamics. Given the unitary evolution

$$
\dot{U}=-i\left[H_{c}+\sum_{j=1}^{n} u_{j} H_{j}\right] U, \quad U(0)=I,
$$

where $H_{c}$ represents a coupling Hamiltonian as in Eq. (69), what is the minimum time required to synthesize any unitary transformation in the coupled spin system, when the control generators $H_{j}$ are local Hamiltonians and are much stronger than the coupling between the spins. Design of time optimal rf-pulse sequences is an important research subject in NMR spectroscopy and quantum information processing as minimizing the time to execute quantum operations can reduce relaxation losses which are always present in an open quantum system as described in the section 2.1. This is the problem of time optimal control of bilinear control systems as in Eq. (2) evolving on compact Lie Groups. The present problem has a special mathematical structure that helps to characterize all the time optimal trajectories [9, 33]. The special mathematical structure manifested in the coupled two spin system motivates a broader study of control systems with the same properties.

The Lie algebra $\mathfrak{g}=s u(4)$ has a decomposition $\mathfrak{g}=\mathfrak{p} \oplus \mathfrak{k}$, where

$$
\mathfrak{k}=-i\left\{I_{\mu}, S_{\nu}\right\}, \quad \mathfrak{p}=-i\left\{I_{\mu} S_{\nu}\right\}
$$

Here $\mathfrak{k}$ is a subalgebra of $\mathfrak{g}$ made from local Hamiltonians. It is easy to verify that

$$
[\mathfrak{k}, \mathfrak{k}] \subset \mathfrak{k}, \quad[\mathfrak{k}, \mathfrak{p}] \subset \mathfrak{p}, \quad[\mathfrak{p}, \mathfrak{p}] \subset \mathfrak{p} .
$$

This decomposition of a real semi-simple Lie algebra $\mathfrak{g}=\mathfrak{p} \oplus \mathfrak{k}$ satisfying (79) is called the Cartan decomposition of the Lie algebra $\mathfrak{g}[34]$.

The coupling Hamiltonian $-i H_{c} \in \mathfrak{p}$ in Eq. (77), while the control Hamiltonians $-i H_{j} \in \mathfrak{k}$. We will assume that the Lie algebra generated by the control terms $-i H_{j}$ span the whole $\mathfrak{k}$, i.e, $\left\{-i H_{j}\right\}_{L A}=\mathfrak{k}$. Under this assumption, a computation shows that the system in Eq. (77) is controllable for any $-i H_{c} \in \mathfrak{p}$. Let $K=\exp (\mathfrak{k})$. We assume that control amplitudes are unbounded apriori and any element of the 
subgroup $K$ of transformations can be synthesized in arbitrary small time. This is typical and will be elaborated in the context of NMR applications and quantum information processing, where any local unitary transformation can be produced in negligible time compared to the evolution of the couplings.

The Cartan decomposition of the Lie algebra $\mathfrak{g}$, in Eq. (79) leads to a decomposition of the Lie group $G$ [34]. Let $\mathfrak{a}$, denote the largest abelian subalgebra contained inside $\mathfrak{p}$. Then, any arbitrary element of the group $G=S U(4)$ can be written as

$$
G=K_{1} \exp \left(a_{1}\right) K_{2}
$$

where $K_{1}, K_{2} \in K$ and $a_{1} \in \mathfrak{a}$. Futhermore, the Cartan decomposition entails that for $-i H_{c} \in \mathfrak{p}$, and $K_{1} \in K$, we have $\underbrace{K_{1}\left(-i H_{c}\right) K_{1}^{\dagger}}_{A d_{K_{1}}\left(-i H_{c}\right)} \in \mathfrak{p}$.

EXAmple 1. For $\mathfrak{g}=s u(4)$, as in Eq. (78), one choice of $\mathfrak{a}$ is

$$
\mathfrak{a}=-i\left\{I_{\alpha} S_{\alpha}\right\} ; \quad \alpha \in\{x, y, z\}
$$

Note $\mathfrak{a}$ is three dimensional. Then, any arbitrary element of any element $U \in S U(4)$ can then be written explicitly as

$$
G=\underbrace{\exp \left(-i \sum_{\mu} c_{\mu} I_{\mu}+\sum_{\nu} d_{\nu} S_{\nu}\right)}_{K_{1}} \exp \left(-i \sum_{\alpha} J_{\alpha} I_{\alpha} S_{\alpha}\right) \underbrace{\exp \left(-i \sum_{\mu} a_{\mu} I_{\mu}+\sum_{\nu} b_{\nu} S_{\nu}\right)}_{K_{2}},
$$

for appropriate choice of coeffecients $a_{\mu}, b_{\nu}, c_{\mu}, d_{\nu}$ and $J_{\alpha}$ etc.

Example 2. For $\mathfrak{g}=s u(n)$ and $\mathfrak{k}=s o(n)$, and $\mathfrak{p}=-i A$, where $A$ is traceless symmetric matrices, the decomposition $\mathfrak{g}=\mathfrak{p} \oplus k$ is a Cartan decomposition. Let $\mathfrak{a}$ be the space of all traceless diagonal matrices, where

$$
\mathfrak{a}=\left\{-i\left[\begin{array}{ccccc}
\lambda_{1} & 0 & 0 & 0 & 0 \\
0 & \lambda_{2} & 0 & 0 & 0 \\
0 & 0 & \ddots & 0 & 0 \\
0 & 0 & 0 & \lambda_{n-1} & 0 \\
0 & 0 & 0 & 0 & \lambda_{n}
\end{array}\right]\right\}
$$

Then any element of $U \in S U(n)$ can be written as $U=K_{1} \exp (D) K_{2}$, where $K_{1}, K_{2} \in$ $S O(n)$ and $D \in \mathfrak{a}$ is a diagonal matrix as above.

Theorem 1. [9] For the control system in Eq. (77), all the elements of $G$, that can be reached starting from $U(0)=I$ in time $T>0$, denoted as $R(I, T)$ is characterized by its closure as

$$
\bar{R}(I, T)=K_{1} \exp \left(T \sum_{j} \alpha_{j} Z_{j}\right) K_{2},
$$


where $K_{1}, K_{2} \in K, \alpha_{j} \geq 0$ and $\sum_{j} \alpha_{j}=1$ and $Z_{j} \in A d_{K}\left(-i H_{c}\right) \cap \mathfrak{a}$. The points $Z_{j}$ are called the Weyl points, the set of points, where the orbit $A d_{K}\left(-i H_{c}\right)$ intersects the Cartan subalgebra a. Let $\mathfrak{c}\left(-i H_{c}\right)$ denote the convex hull of the Weyl points $A d_{K}\left(-i H_{c}\right) \cap \mathfrak{a}$, then the reachable set can also be written as

$$
\bar{R}(I, T)=K_{1} \exp \left(T \mathfrak{c}\left(-i H_{c}\right)\right) K_{2} .
$$

REMARK 1. In essence, the KAK decomposition of the group $G$ allows us to write any $U \in G$ as $U=K_{1} \exp (Y) K_{2}$ with $Y \in \mathfrak{a}$ and the minimum time $T$ to synthesize $U$ is to find the smallest time $T$, such that $Y / T$ lies in the convex hull of the Weyl points $Z_{j}$. Given that $T$ is the minimum time such that $Y / T=\sum_{j} \alpha_{j} \underbrace{A d_{K_{j}}\left(-i H_{c}\right)}_{Z_{j}}$, with $\alpha_{j}>0, \sum_{j} \alpha_{j}=1$ and $\underbrace{A d_{K_{j}}\left(-i H_{c}\right)}_{Z_{j}} \in \mathfrak{a}$, we can synthesize $\exp (Y)$ as

$$
\exp (Y)=\prod_{j=1}^{n+1} K_{j} \exp \left(-i H_{c} t_{j}\right) K_{j}^{\dagger},
$$

where $K_{j} \in K$, and therefore take negligible time to synthesize. The optimal trajectory consists of a sequence of fast control rotations, interspersed with the periods of free evolution.

EXAmple 3. In example 2, the Weyl points $Z_{j}$ are

$$
-i\left[\begin{array}{ccccc}
\lambda_{\sigma(1)} & 0 & 0 & 0 & 0 \\
0 & \lambda_{\sigma(2)} & 0 & 0 & 0 \\
0 & 0 & \ddots & 0 & 0 \\
0 & 0 & 0 & \lambda_{\sigma(n-1)} & 0 \\
0 & 0 & 0 & 0 & \lambda_{\sigma(n)}
\end{array}\right],
$$

the various permutations of the eigenvalues of $H_{c}$. The closure of the reachable set in time $T$ is all matrices of the form

$$
K_{1} \exp \left(-i\left[\begin{array}{ccccc}
\mu_{1} & 0 & 0 & 0 & 0 \\
0 & \mu_{2} & 0 & 0 & 0 \\
0 & 0 & \ddots & 0 & 0 \\
0 & 0 & 0 & \mu_{n-1} & 0 \\
0 & 0 & 0 & 0 & \mu_{n}
\end{array}\right]\right) K_{2}
$$

where $\mu=\left(\mu_{1}, \mu_{2}, \ldots, \mu_{n}\right)^{\prime}$ satisfies $\mu \prec T \lambda$, where $\lambda=\left(\lambda_{1}, \lambda_{2}, \ldots, \lambda_{n}\right)^{\prime}$, (the symbol $\prec$ stands for majorization), i.e., $\mu$ lies in convex hull of the vector $T \lambda$ and its various permutations. 
EXAMPLE 4. For the coupled spins, as in example 1, if we choose $\mathfrak{a}=-i\left\{I_{\alpha} S_{\alpha}\right\}$, then the Weyl points $Z_{j}$ have the form $c_{x} I_{x} S_{x}+c_{y} I_{y} S_{y}+c_{z} I_{x} S_{x}$, where

$$
\left\{\left(c_{x}, c_{y}, c_{z}\right) \in\left\{\epsilon_{1} c_{\sigma(1)}, \epsilon_{2} c_{\sigma(2)}, \epsilon_{3} c_{\sigma(3)} \mid \epsilon_{i}= \pm 1, \prod_{i} \epsilon_{i}=1\right\},\right.
$$

where $\left(c_{\sigma(1)}, c_{\sigma(2)}, c_{\sigma(3)}\right)$ are various permutations of $\left(c_{1}, c_{2}, c_{3}\right)$, where $c_{1} \geq c_{2} \geq\left|c_{3}\right|$. Then the reachable set for the system in time $T$ in example 1 is now described by Theorem (1) and can be explicitly written as

$$
\bar{R}(I, T)=K_{1} \exp \left(b_{1} I_{x} S_{x}+b_{2} I_{y} S_{y}+b_{3} I_{z} S_{z}\right) K_{2}
$$

such that $b_{1} \geq b_{2} \geq\left|b_{3}\right|$, and $b_{1} \leq T c_{1}$ and $b_{1}+b_{2} \pm\left|b_{3}\right| \leq T\left(c_{1}+c_{2} \pm\left|c_{3}\right|\right)$. As before $K_{1}, K_{2} \in K=S U(2) \otimes S U(2)$.

Theorem 1, gives a complete characterization of the reachable set for coupled qubit system. The results derive from the Cartan decomposition of the group $G=$ $S U(4)$ in terms of the associated subgroup $K=S U(2) \otimes S U(2)$, where elements of $K$ can be synthesized in arbitrarily small time. Until now we have only talked about coupled two qubits. Experiments in Quantum information processing and NMR spectroscopy involve control of dynamics of multiple coupled spins. For a system of $n$ spin $\frac{1}{2}$, the Hilbert space is $2^{n}$ dimensional. Unitary transformation on such a space belongs to the group $G=S U\left(2^{n}\right)$. The control Hamiltonians for such a system generate the subgroup $K=S U(2) \otimes S U(2) \ldots S U(2)$, the group of local unitary transformations that effects individual spins. Lie group decompositions such as the $K A K$ decomposition can be used to decompose any unitary transformation $U \in G$ as

$$
U=K_{n+1} \exp \left(-i H_{c} t_{n}\right) \ldots \exp \left(-i H_{c} t_{1}\right) K_{1},
$$

where $K_{i} \in K$ are local rotations and are interspersed with evolution of the coupling Hamiltonian $-i H_{c}$ for appropriate time. These decompositions then provide explicit synthesis methods for generating unitary transformations in $G$.

There are numerous beautiful control problems of efficient synthesis of unitary transformations belonging to $S U\left(2^{n}\right)$, using the coupling Hamiltonian between the spins and the control subgroup $K$ [10]. Finding time optimal control for synthesizing unitary transformations in the big space $G$ can be reduced to problems in Sub-riemannian goemetry and have been recently studied [29]. Characterization of time optimal trajectories for multiple spin systems however remains largely open.

\section{Control of Coupled Spin Dynamics in the Presence of Relaxation.}

Many experiments in coherent spectroscopy and quantum information processing require transfer between different states of coupled spin system. Presence of decoherence arising due to coupling to the environment, limits how close the state of a spin system can be driven to a target state. In the previous section, we described problems in 


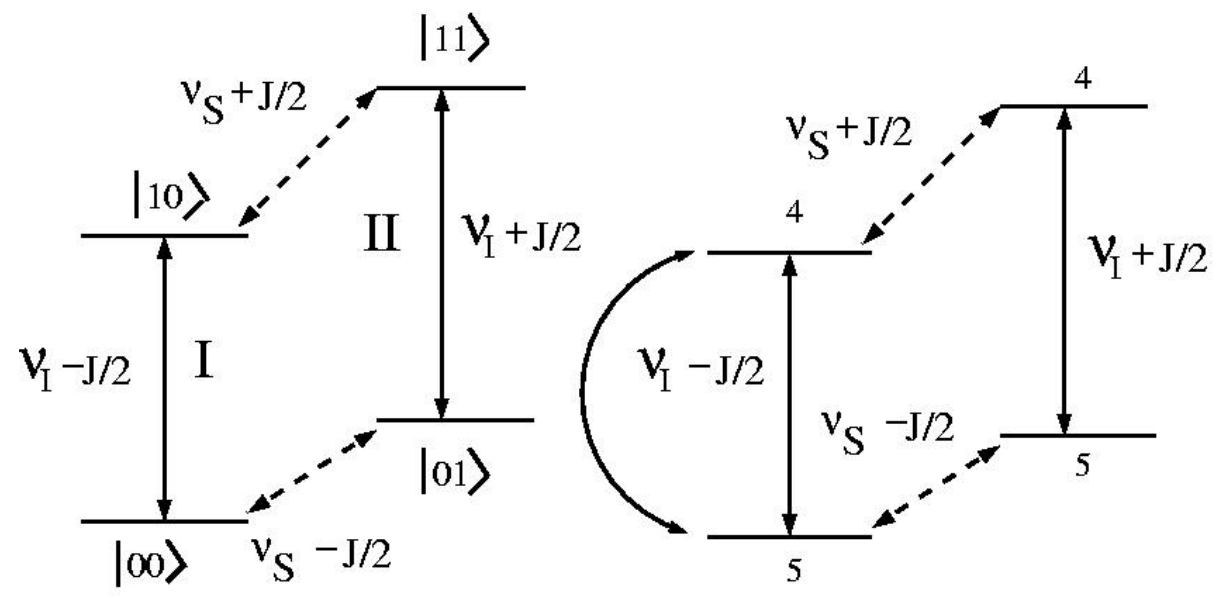

(A)

FIG. 7. The figure $A$ shows the eigenstates of the Hamiltonian $H_{0}+H_{c}$ for the two spin system as in Eq. (86). The energies are in the frequency units. Figure B corresponds to an ensemble where there are excess of spin $I$ oriented along the $B_{0}$ field direction. The populations in various states are shown below the energy bars.

time optimal control of coupled spin dynamics with the goal of minimizing decoherence effects by reducing the time to perform quantum operations. In this section, we describe some problems of optimal design of trajectories of coupled spin evolution so that they suffer minimum decoherence loss. We show that by exploiting explicit models for decoherence, represented by Lindblad operators as described in Eq. (33), it is possible to design trajectories of the coupled spin system so that they suffer minimum decoherence loss [30, 31, 32].

We consider a coupled spin system consisting of spin $I$ and $S$. The Hamiltonian for the spin system takes the form

$$
H(t)=\underbrace{2 \pi \nu_{I} I_{z}+2 \pi \nu_{S} S_{z}}_{H_{0}}+\underbrace{\pi J 2 I_{z} S_{z}}_{H_{c}}+\underbrace{2 \pi A \cos (\omega t+\theta(t))}_{u_{1}(t)} \underbrace{\left(I_{x}+S_{x}\right)}_{H_{1}}
$$

The first two terms of $H_{0}$ represent energy of the spins $I$ and $S$ in a static magnetic field along $z$ direction. The term $2 I_{z} S_{z}$, corresponds to interaction Hamiltonian, which gives a positive contribution when spins are oriented alike and negative contribution when the spins are oriented opposite to each other. The control consists of an oscillating magnetic field along the $x$ direction, whose amplitude, frequency and phase, given by $A(t), \omega$ and $\phi$ can be varied. In these experiments, $J \ll A \ll \nu_{I}, \nu_{S}$. Typical values of $J$ and $A$ are in $\mathrm{Hz}$ and Khz respectively, while $\nu_{I}$ and $\nu_{S}$ at $B_{0}$ field strength of Tesla is 100 of MHz. 
The eigenstate of the Hamiltonian are the product operator basis $|00\rangle,|01\rangle,|10\rangle$ and $|11\rangle$, where $|0\rangle$ and $|1\rangle$ are eigenstates of the $\sigma_{z}$, with eigenvalues $\frac{1}{2}$ and $-\frac{1}{2}$. Therefore, the energies of these eigenstates are $-\frac{\left(\nu_{I}+\nu_{S}-J / 2\right)}{2}, \frac{\left(\nu_{I}-\nu_{S}-J / 2\right)}{2}, \frac{\left(-\nu_{I}+\nu_{S}-J / 2\right)}{2}$ and $\frac{\left(\nu_{I}+\nu_{S}+J / 2\right)}{2}$. These energies are depicted in the energy level diagram in Fig. 7A. Observe, that the difference in the energies of the $|1\rangle$ and 0$\rangle$ states of spin $I$ depends on whether the $S$ spin is in $|0\rangle$ or $|1\rangle$ state and corresponds to transitions $I$ and $I I$ in Fig. 7A. The corresponding energies are $\nu_{I}-\frac{J}{2}$ and $\nu_{I}+\frac{J}{2}$. Therefore, if one performs an NMR experiment as described earlier in Fig. 3 on an spin ensemble of coupled spin $I$ and $S$ where $I$ spins have the Larmor frequency $\omega_{I}$, then one observes two resonances, one at $\nu_{I}-\frac{J}{2}$ and $\nu_{I}+\frac{J}{2}$.

Fig. 7B, shows the state of a spin ensemble with population in different states written below the energy bar. The proportion of the ensemble when spin $I$ in the state $|0\rangle$, vs when the spin $I$ is in the state $|1\rangle$, is $5 / 4$. While the ensemble has equal number of spin $S$ in $|0\rangle$ and $|0\rangle$ states. Writing down a density matrix for this system then gives,

$$
\rho=\frac{5}{18}|00\rangle\left\langle 00\left|+\frac{5}{18}\right| 01\right\rangle\left\langle 01\left|+\frac{4}{18}\right| 10\right\rangle\left\langle 10\left|+\frac{4}{18}\right| 11\right\rangle\langle 11| .
$$

We obtain

$$
\rho=\frac{1}{4} \mathbf{1}+\frac{1}{18} I_{z}
$$

which signifies that we have an ensemble of spins with an excess of spins $I$ oriented along the $z$ direction. An important experiment in NMR spectroscopy is to synthesize unitary transformations that will transform an ensemble of the kind

$$
\frac{1}{4} \mathbf{1}+\alpha_{I} I_{z}+\alpha_{S} S_{z}
$$

where $\alpha_{I}>\alpha_{S}$ into an ensemble that looks like $\frac{1}{4} \mathbf{1}+\alpha_{S} I_{z}+\alpha_{I} S_{z}$. If the gyromagnetic ratio $\gamma_{I}>\gamma_{S}$, then in thermal equilibrium, spins $I$ are more polarized than spin $S$ and therefore $\alpha_{I}>\alpha_{S}$. By transforming the ensemble so that more of the spins $S$ get more polarized compared to their equilibrium state, it is possible to improve the sensitivity of NMR experiments that determine the Larmor frequency of spins $S$. This experiment is called, transfer of polarization experiment. To make matters more transparent, we assume $\alpha_{S}=0$ and drop the factor $\frac{1}{4} \mathbf{1}$, as this part of the density matrix doesnot transform under rotations. We consider operations that will transform the spin ensemble from the initial state

$$
I_{z} \rightarrow S_{z}
$$

One method for performing this manipulation is to first perform a rotation on spin $I$ conditioned on the state of spin $S$, so that the $|10\rangle \leftrightarrow|00\rangle$, while $|01\rangle$ and $|11\rangle$ is 
unperturbed. In the language of quantum information processing, this is so called a controlled not (CNOT) operation, and the corresponding unitary transformation, denoted $U_{\text {cnot }}$ inverts the state of spin $I$, conditioned on state of spin $S$ being $|0\rangle$. This is depicted by arc $I$ in Fig. 7 B. Now, we can perform a controlled not operation on spin $S$, such that the $S$ spin is inverted if spin $I$ is 0 . As a result of first controlled not operation, the ensemble in (87) transforms to

$$
\rho=\frac{5}{18}|10\rangle\left\langle 10\left|+\frac{5}{18}\right| 01\right\rangle\left\langle 01\left|+\frac{4}{18}\right| 00\right\rangle\left\langle 00\left|+\frac{4}{18}\right| 11\right\rangle\langle 11|=\frac{1}{4} \mathbf{1}+\frac{1}{18} 2 I_{z} S_{z} .
$$

As a result of second controlled not operation, the ensemble in (89) transforms to

$$
\rho=\frac{5}{18}|10\rangle\left\langle 10\left|+\frac{5}{18}\right| 00\right\rangle\left\langle 00\left|+\frac{4}{18}\right| 01\right\rangle\left\langle 01\left|+\frac{4}{18}\right| 11\right\rangle\langle 11|=\frac{1}{4} \mathbf{1}+\frac{1}{18} S_{z} .
$$

Another operation of fundamental importance in quantum information processing is to transform a separable state $|00\rangle$ to an entangled state of the form $\frac{|00\rangle+|11\rangle}{2}$. Entangled states are useful resources in many quantum information processing protocols. One mechanism of performing such an operation is to start with an initial state $|00\rangle$ and transform it as

$$
|00\rangle \rightarrow|0\rangle \frac{|0\rangle+|1\rangle}{\sqrt{2}}
$$

Such a transformation simply involves doing a local unitary transformation of the type $\exp \left(-i \frac{\pi}{4} \mathbf{1} \otimes \sigma_{x}\right)$ and can be obtained by evolution of local Hamiltonians as described in the previous section. Such operations are significantly faster than the evolution of the coupling Hamiltonians. Now, by performing the unitary transformation, $U_{\text {cnot }}$ on this state, where the state of the first spin $I$ is inverted, conditioned on the state of the spin $S$, such that $|00\rangle \rightarrow|00\rangle$ and $|01\rangle \rightarrow|11\rangle$, we obtain

$$
|0\rangle \frac{|0\rangle+|1\rangle}{\sqrt{2}} \rightarrow \frac{|00\rangle+|11\rangle}{2} .
$$

In the presence of decoherence or dissipation in the system, the desired transfer cannot be performed with complete fidelity. Interesting optimal control problems arise with the goal of maximizing the fidelity of the desired transformations in the presence of decoherence as described subsequently. The control system describing the transfer is obtained by first writing the Schröedinger equation of the coupled spin system in terms of the product basis $|00\rangle,|01\rangle,|10\rangle$ and $|11\rangle$. This gives us that 
$\begin{aligned} \text { (91) } & \frac{d}{d t}\left[\begin{array}{l}\psi_{1} \\ \psi_{2} \\ \psi_{3} \\ \psi_{4}\end{array}\right] \\ & =\frac{-i}{2}\left[\begin{array}{cccc}-\omega_{I}-\omega_{S}+J & u & u & 0 \\ u & -J+\omega_{S}-\omega_{I} & 0 & u \\ u & 0 & \omega_{I}-\omega_{S}+J & u \\ 0 & u & u & \omega_{I}+\omega_{S}+J\end{array}\right]\left[\begin{array}{l}\psi_{1} \\ \psi_{2} \\ \psi_{3} \\ \psi_{4}\end{array}\right] .\end{aligned}$

We add decoherence into our system model by introducing fluctuations into the system Hamiltonian $H(t)$ in Eq. (91). The resulting density matrix equation then takes the form

$$
\dot{\rho}=-i\left[H(t)+f_{1}(t) I_{z}+f_{2}(t) S_{z}+f_{3}(t) I_{z} S_{z}, \rho\right]
$$

Where $f_{1}(t), f_{2}(t)$ and $f_{3}(t)$ are assumed to be uncorrelated fluctuations such that $E\left[f_{i}(t+\tau) f_{j}(t)\right]=\delta_{i j} \delta(\tau) k_{i}$. This captures the fact that various terms contributing to the Hamiltonian fluctuate. This leads to the master equation

$$
\dot{\rho}=-i[H(t), \rho]+\underbrace{k_{1}\left[i I_{z}\left[i I_{z}, \rho\right]+k_{2}\left[i S_{z}\left[i S_{z}, \rho\right]+k_{3}\left[i I_{z} S_{z}\left[i I_{z} S_{z}, \rho\right]\right.\right.\right.}_{L(\rho)} .
$$

Now, by choosing $u(t)=2 \cos \omega_{I} t$, where $\omega_{I}$ is the resonance frequency of spin $I$, and transforming into a rotating frame described by taking the density matrix

$$
\rho(t) \rightarrow \exp \left(i H_{0} t\right) \rho(t) \exp \left(-i H_{0} t\right)
$$

we obtain that

$$
\dot{\rho}=-i[2 J I_{z} S_{z}+\underbrace{A \cos \phi(t)}_{u(t)} I_{x}+\underbrace{A \sin \phi(t)}_{v(t)} I_{y}, \quad \rho]+L(\rho) .
$$

We can rewrite the corresponding density equation as

$$
\frac{d}{d t}\left[\begin{array}{c}
z_{1} \\
y_{1} \\
x_{1} \\
x_{2} \\
y_{2} \\
z_{2}
\end{array}\right]=\left[\begin{array}{cccccc}
0 & u & -v & 0 & 0 & 0 \\
-u & -k & -J & 0 & 0 & 0 \\
v & J & -k & 0 & 0 & 0 \\
0 & 0 & 0 & -k & -J & v \\
0 & 0 & 0 & J & -k & -u \\
0 & 0 & 0 & -v & u & 0
\end{array}\right]\left[\begin{array}{c}
z_{1} \\
y_{1} \\
x_{1} \\
x_{2} \\
y_{2} \\
z_{2}
\end{array}\right]
$$

where $\left(x_{1}, y_{1}, z_{1}\right)$ is a Bloch vector associated with the two level system $|00\rangle$ and $|01\rangle$. Similarly, $\left(x_{2}, y_{2}, z_{2}\right)$ is the Bloch vector associated with the two level system $|10\rangle$ and $|11\rangle$. 
ON SOME MODEL PROBLEMS IN QUANTUM CONTROL

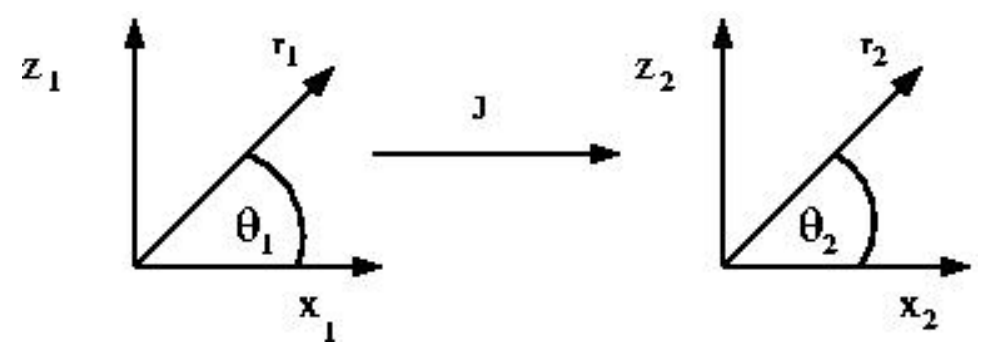

FIG. 8. The figure shows pictorially the states $X_{1}, X_{2}, Z_{1}, Z_{2}$, as in Eq. (96), where the angle $\theta_{1}$ and $\theta_{2}$ can be controlled arbitrarily fast.

The goal is then to synthesize $u(t)$ and $v(t)$ that transfer

$$
\left[\begin{array}{l}
1 \\
0 \\
0 \\
0 \\
0 \\
1
\end{array}\right] \rightarrow\left[\begin{array}{c}
-1 \\
0 \\
0 \\
0 \\
0 \\
1
\end{array}\right]
$$

This would correspond to the selective inversion of the transition $I$ in the Fig. 7A.

If we re-express the above equations with coordinates $Z_{1}=\frac{z_{1}+z_{2}}{2}$ and $Z_{2}=\frac{z_{1}-z_{2}}{2}$. Similarly, we define $X_{1}, X_{2}, Y_{1}, Y_{2}$. Then, we obtain the following control system,

$$
\frac{d}{d t}\left[\begin{array}{c}
Z_{1} \\
X_{1} \\
X_{2} \\
Z_{2}
\end{array}\right]=\left[\begin{array}{cccc}
0 & -u(t) & 0 & 0 \\
u(t) & -k & -J & 0 \\
0 & J & -k & v(t) \\
0 & 0 & -v(t) & 0
\end{array}\right]\left[\begin{array}{c}
Z_{1} \\
X_{1} \\
X_{2} \\
Z_{2}
\end{array}\right]
$$

Now, the goal is to steer the above system from $(1,0,0,0)^{\prime}$ to $(0,0,0, \eta)^{\prime}$, maximizing the value of $\eta$. Here the parameters $J$ and $k$ represents the coupling and relaxation in the system. Eq. (96) represents a typical problem in the control of quantum systems in the presence of decoherence where one requires natural dynamics, represented by the parameter $J$ to steer the system between points of interest and the natural dynamics is dissipative, as represented by the parameter $k$. When the strength $J$ is comparable to the parameter $k$, one necessarily dissipates. resulting in $\eta<1$. Since the controls can be made much larger than the natural parameters in the system, we define $r_{1}=$ $\sqrt{Z_{1}^{2}+X_{1}^{2}}, r_{2}=\sqrt{Z_{1}^{2}+X_{1}^{2}}, \tan \theta_{1}=\frac{Z_{1}}{X_{1}}$ and $\tan \theta_{2}=\frac{Z_{2}}{X_{2}}$. Writing an equation for $r_{1}$ and $r_{2}$, gives us

$$
\frac{d}{d t}\left[\begin{array}{l}
r_{1} \\
r_{2}
\end{array}\right]=\left[\begin{array}{cc}
-k u_{1}^{2} & -J u_{1} u_{2} \\
J u_{1} u_{2} & -k u_{2}^{2}
\end{array}\right]
$$


where $u_{1}(t)=\cos \theta_{1}(t)$ and $u_{2}(t)=\cos \theta_{2}(t)$. The goal is that for $0 \leq u_{1}(t), u_{2}(t) \leq 1$, find the maximum possible transfer to the final state $r_{2}$, starting from the initial state $\left(r_{1}, r_{2}\right)=(1,0)$. Now, this problem can be solved by direct application of the maximum principle.

Let $\left(\lambda_{1}, \lambda_{2}\right)$, represent the costate variable for the system in Eq. (97). Along the optimal trajectory, the Hamiltonian

$$
H\left(u_{1}, u_{2}\right)=\left[\begin{array}{ll}
\lambda_{1} & \lambda_{2}
\end{array}\right]\left[\begin{array}{cc}
-k u_{1}^{2} & -J u_{1} u_{2} \\
J u_{1} u_{2} & -k u_{2}^{2}
\end{array}\right]\left[\begin{array}{l}
r_{1} \\
r_{2}
\end{array}\right]
$$

should be maximized. The Hamiltonian can then be written as

$$
H\left(u_{1}, u_{2}\right)=\left[\begin{array}{ll}
u_{1} & u_{2}
\end{array}\right] \underbrace{\left[\begin{array}{cc}
-k \lambda_{1} r_{1} & J \frac{\lambda_{2} r_{1}-\lambda_{1} r_{2}}{2} \\
J \frac{\lambda_{2} r_{1}-\lambda_{1} r_{2}}{2} & -k \lambda_{2} r_{2}
\end{array}\right]}_{B}\left[\begin{array}{l}
u_{1} \\
u_{2}
\end{array}\right] .
$$

Then the optimal $\left(u_{1}^{*}, u_{2}^{*}\right)$ should satisfy that $H\left(u_{1}^{*}, u_{2}^{*}\right)=0$. This then implies that $\operatorname{det} B=0$ and $B\left[\begin{array}{l}u_{1}^{*} \\ u_{2}^{*}\end{array}\right]=0$. Then substituting $\operatorname{det} B=0$ and letting $a=\frac{\lambda_{2}}{\lambda_{1}}, b=\frac{r_{2}}{r_{1}}$ and $\xi=\frac{k}{J}$, we obtain that

$$
\sqrt{\frac{b}{a}}=\sqrt{1+\xi^{2}}-\xi
$$

Now, using the condition that $B\left[\begin{array}{l}u_{1}^{*} \\ u_{2}^{*}\end{array}\right]=0$, implies that $\frac{u_{1}^{*}}{u_{2}^{*}}=\frac{a-b}{2 \xi}$, resulting in

$$
\frac{u_{2}^{*} r_{2}}{u_{1}^{*} r_{1}}=\sqrt{1+\xi^{2}}-\xi
$$

The optimal feedback control law entails for the equation (96), that $\frac{X_{2}}{X_{1}}=\sqrt{1+\xi^{2}}-\xi$. This policy leads to an optimal value of $\eta=\sqrt{1+\xi^{2}}-\xi$ in (96) and this is the largest possible value of $r_{2}$ in (98). Infact, it is now straightforward to write down an optimal return function $V\left(r_{1}, r_{2}\right)$, representing the maximum possible achievable value of $r_{2}$ starting from arbitrary value of $r_{1}$ and $r_{2}$ and it turns out to be

$$
V\left(r_{1}, r_{2}\right)=\sqrt{\eta^{2} r_{1}^{2}+r_{2}^{2}}
$$

In Eq. (92), we assumed that fluctuations $f_{i}(t)$ are uncorrelated. Interesting model systems arise when we consider correlations between various noise mechanisms [31]. Suppose we assume that $E\left(f_{1}(t) f_{3}(t+\tau)\right)=k_{c} \delta(\tau)$, then the following transfer problem arises which is of fundamental interest in NMR spectroscopy ([31]). Given the control system 


\section{Transfer Efficiency}

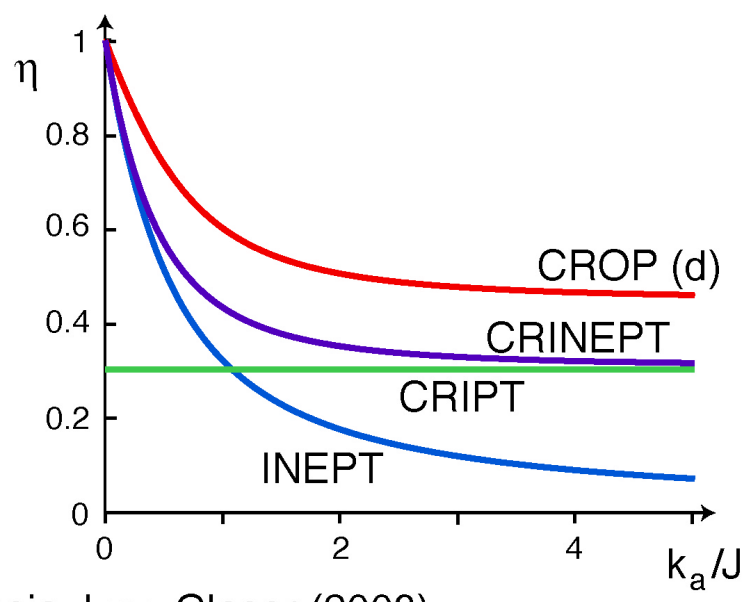

(d) Khaneja, Luy, Glaser (2003)

FIG. 9. The figure shows efficiency of various state of the art pulse sequences as a function of $\frac{k_{a}}{J}$ for the transfer in Eq. (99) for $k_{c}=.75$. The CROP pulse sequences developed using optimal control of system in Eq. (99) provide the optimal transfer.

$$
\frac{d}{d t}\left[\begin{array}{c}
Z_{1} \\
Y_{1} \\
X_{1} \\
X_{2} \\
Y_{2} \\
Z_{2}
\end{array}\right]=\left[\begin{array}{cccccc}
0 & u(t) & -v(t) & 0 & 0 & 0 \\
-u(t) & -k_{a} & -J & 0 & k_{c} & 0 \\
v(t) & J & -k_{a} & k_{c} & 0 & 0 \\
0 & 0 & k_{c} & -k_{a} & -J & v(t) \\
0 & k_{c} & 0 & J & -k_{a} & -u(t) \\
0 & 0 & 0 & -v(t) & u(t) & 0
\end{array}\right]\left[\begin{array}{c}
Z_{1} \\
Y_{1} \\
X_{1} \\
X_{2} \\
Y_{2} \\
Z_{2}
\end{array}\right]
$$

find optimal $(u(t), v(t))$ such that starting from $\left(Z_{1}, Y_{1}, X_{1}, X_{2}, Y_{2}, Z_{2}\right)=(1,0,0,0,0$, $0)$, what is the largest value of $(0,0,0,0,0, \eta)$.

The above optimal control problem can be solved in closed form. Consider the vectors $l_{2}=\left(X_{2}, Y_{2}\right)$ and $l_{1}=\left(X_{1}, Y_{1}\right)$. The optimal solution is then given by the following two invariants of motion. The ratio

$$
\frac{l_{2}}{l_{1}}=\sqrt{1+\xi^{2}}-\xi=\eta ; \quad \xi=\sqrt{\frac{k_{a}^{2}-k_{c}^{2}}{k_{a}^{2}+J^{2}}}
$$

is maintained constant and the angle between vectors $l_{2}$ and $l_{1}$ is maintained constant. The maximum transfer of efficiency is then $\eta$.

It is worthwhile to point out that researchers in magnetic resonance have developed novel pulse sequences that have improved the transfer described in Eq. (99), 
however the fundamental limits of the transfer described here was not known. Fig. 9 shows plot of transfer efficiency of various state of the art pulse sequences as a function of the ratio $\frac{k_{a}}{J}$ for $k_{c}=.75$. The CROP pulse sequence obtained by solving the above transfer problem using methods of optimal control performs better than all state of the art methods and provide significant improvement is sensitivity. Furthermore methods of optimal control help to provide limits on how close can a quantum dynamical system be driven to a target state.

In this section we provided some concrete examples of state transfer problems involving control of coupled spin dynamics in the presence of decoherence or relaxation. We showed how optimal control of these dissipative bilinear systems can lead to design of better experiments. A systematic study of the controllability and optimal control problems related to Lindblad equations of open quantum systems is expected to have immediate impact in areas of coherent spectroscopy and quantum information processing.

6. Conclusion. In this paper, we presented some model problems that arise in the control of spin dynamics in experiments in magnetic resonance. Due to the space limitation, an important class of quantum control problems related to the realtime monitoring of state of the quantum system and the backaction of these measurements on the state of the system have not been discussed [36]. The control issues discussed in this article are ubiquitous in problems involving control of quantum mechanical systems as encountered in various areas of physics and chemistry. Although experiments in spectroscopy involving probing and control of quantum mechanical systems using electromagnetic fields are now more than 50 years old, sophisticated design and shaping of the electromagnetic control fields only began to emerge in $80^{\prime} s$. Around then physicists and chemists started to think along traditional control related issues of controllability and optimal control. The intellectual program initiated by Roger Brockett in 1970, on the study of nonlinear control systems and the use of differential geometric methods to address these problems is foundational for the subject of quantum control.

\section{REFERENCES}

[1] R. R. Ernst, G. Bodenhausen, and A. Wokaun, Principles of Nuclear Magnetic Resonance in One and Two Dimensions, Clarendon Press, Oxford, 1987.

[2] C. P. Slichter, Principles of Magnetic Resonance, Springer Verlag 1978.

[3] A. Abragam, The Principles of Nuclear Magnetism Oxford University Press, 1978.

[4] K. Wüthrich, NMR of Proteins and Nucleic Acids, Wiley \& Sons, 1986.

[5] J. Cavanagh, W. J. Fairbrother, A. G. Palmer, and N. J. Skelton, Protein NMR Spectroscopy, Principles and Practice, Academic Press, 1996.

[6] R. W. Brockett, System Theory on Group Manifolds and Coset Spaces, SIAM Journal of Control, 10(1972), pp. 265-284. 
[7] V. Jurdjevic and H. Sussmann, Control Systems on Lie Groups, Journal of Differential Equations, 12(1972), pp. 313-329.

[8] R. W. Brockett, Nonlinear Control Theory and Differential Geometry, Proceedings of the International Congress of Mathematicians, 1357-1367, 1984.

[9] N. Khaneja, R.W. Brockett, and S.J. Glaser, Time optimal control of spin systems, Phys. Rev. A 63(2001), 032308.

[10] N. Khaneja And S.J. Glaser, Cartan decomposition of $S U\left(2^{n}\right)$ and control of spin systems, Chemical Physics, 267(2001), pp. 11-23.

[11] D. D'Alessandro, Constructive controllability of one and two spin 1/2 particles, Proceedings 2001 American Control Conference, Arlington, Virginia, June 2001.

[12] V. Ramakrishna, H. Rabitz, M. V. Salapaka, M. Dahleh, and A. Peirce, Controllability of the Molecular Systems, Phys. Rev. A, 51(1995), pp. 960-66.

[13] A. G. Redfield, The theory of relaxation processes, Adv. Magn. Reson. 1(1965), pp. 1-32.

[14] G. Lindblad, On the generators of quantum dynamical semigroups, Commun. Math. Phys. 48(1976), 199.

[15] H. J. Metcalf and P. Straten, Laser Cooling and Trapping, Springer, 1999.

[16] S. E. Sklarz, D. J. Tannor, and N. Khaneja, Optimal control of quantum dissipative dynamics: analytic solution for cooling a three level Lambda system, Phys. Rev. A, 69(2004), 053408 .

[17] M. H. LevitT, Prog. NMR Spectrosc, 18(1986), 61.

[18] M. S. Silver, R. I. Joseph, C.-N. Chen, V. J. Sank, And D. I. Hoult, Nature. 310(1984), 681.

[19] David E Rourke, Ph.D. Thesis, 1992.

[20] M. Shinnar, S. Eleff, H. Subramanian, and J. S. Leigh, Resonance Med. 12(1989), pp. $74-80$.

[21] P. Le Roux, Proc. 7th SMRM 1049, 1988.

[22] J. S. Li And N. Khaneja, Control of inhomogeneous quantum ensembles, Phys. Rev. A, 73(2006), 030302.

[23] J. S. Li And N. KhaneJa, Ensemble controllability of the Bloch equations, IEEE Conference on Decision and Control, San-Diego, 2006.

[24] J. S. LI, Control of Inhomogeneous Ensembles, Ph.d. Thesis, Harvard University, 2006.

[25] B. Pryor And N. Khaneja, Fourier synthesis techniques for control of inhomogeneous quantum systems, IEEE Conference on Decision and Control, San Diego, December 2007.

[26] T.E. Skinner, T. Reiss, B. Luy, N. Khaneja, and S.J. Glaser, J. Magn. Reson., 163(2003), 8.

[27] K. Kobzar, B. Luy, N. Khaneja, and S. J. Glaser, Pattern Pulses: design of arbitrary excitation profiles as a function of pulse amplitude and offset, J. Magn. Reson. 173(2005), pp. 229-235.

[28] K. Kobzar, T. E. Skinner, N. Khaneja, S. J. Glaser, and B. Luy, Exploring the limits of broadband excitation and inversion pulses, J. Magn. Reson. 170(2004), pp. 236-243.

[29] N. Khaneja, S. J. Glaser, and R. W. Brockett, Sub-Riemannian geometry and optimal control of three spin systems, Phys. Rev. A, 65(2002), 032301.

[30] N. Khaneja, T. Reiss, B. Luy, and S. J. Glaser, Optimal control of spin dynamics in the presence of relaxation, J. Magn. Reson. 162(2003), pp. 311-319.

[31] N. Khaneja, B. Luy, And S.J. Glaser, Boundary of quantum evolution under decoherence, Proceedings of National Academy of Sciences, 100:23(2003), pp. 13162-66.

[32] N. Khaneja, Jr. Shin Li, C. Kehlet, B. Luy, and S.J. Glaser, Broadband relaxation optimized polarization transfer in magnetic resonance, Proceedings of National Academy of Sciences, USA. 101(2004), pp. 14742-47.

[33] H. YuAn And N. Khaneja, Reachable sets of bilinear control system with time varying drift, 
System and Control Letters, 55(2006), 501.

[34] S. Helgason, Differential Geometry, Lie Groups, and Symmetric Spaces , Academic Press, 1978.

[35] M. Nielsen and I. Chuang, Quantum Information and Computation, Cambridge University Press, 2000.

[36] H. Mabuchi and N. Khaneja, Principles and applications of control in quantum systems, International Journal of Robust and Nonlinear Control, 15(2005), pp. 647-667. 